\title{
FAST EIGENPAIRS COMPUTATION WITH OPERATOR ADAPTED WAVELETS AND HIERARCHICAL SUBSPACE CORRECTION*
}

\author{
HEHU XIE ${ }^{\dagger}$, LEI ZHANG ${ }^{\ddagger}$, AND HOUMAN OWHADI ${ }^{\S}$
}

\begin{abstract}
We present a method for the fast computation of the eigenpairs of a bijective positive symmetric linear operator $\mathcal{L}$. The method is based on a combination of operator adapted wavelets (gamblets) with hierarchical subspace correction. First, gamblets provide a raw but fast approximation of the eigensubspaces of $\mathcal{L}$ by block-diagonalizing $\mathcal{L}$ into sparse and well-conditioned blocks. Next, the hierarchical subspace correction method computes the eigenpairs associated with the Galerkin restriction of $\mathcal{L}$ to a coarse (low-dimensional) gamblet subspace and then corrects those eigenpairs by solving a hierarchy of linear problems in the finer gamblet subspaces (from coarse to fine, using multigrid iteration). The proposed algorithm is robust to the presence of multiple (a continuum of) scales and is shown to be of near-linear complexity when $\mathcal{L}$ is an (arbitrary local, e.g., differential) operator mapping $\mathcal{H}_{0}^{s}(\Omega)$ to $\mathcal{H}^{-s}(\Omega)$ (e.g., an elliptic PDE with rough coefficients).
\end{abstract}

Key words. multiscale eigenvalue problem, gamblet decomposition, multigrid iteration, subspace correction, numerical homogenization

AMS subject classifications. 65N30, 65N25, 65L15, 65B99

DOI. $10.1137 / 18 \mathrm{M} 1194079$

1. Introduction. Solving large scale eigenvalue problems is one of the most fundamental and challenging tasks in modern science and engineering. Although high-dimensional eigenvalue problems are ubiquitous in physical sciences, data and imaging sciences, and machine learning, the class of eigensolvers is not as diverse as that of linear solvers (which comprises many efficient algorithms such as geometric and algebraic multigrid [11, 20], approximate Gaussian elimination [33], etc.). In particular, eigenvalue problems may involve operators with nonseparable multiple scales, and the nonlinear interplay between those coupled scales and the eigenvalue problem poses significant challenges for numerical analysis and scientific computing $[4,14,59,37]$.

Krylov subspace type methods remain the most reliable and efficient tools for large scale eigenproblems, and alternative approaches such as optimization based methods

*Received by the editors June 13, 2018; accepted for publication (in revised form) August 14, 2019; published electronically November 5, 2019.

https://doi.org/10.1137/18M1194079

Funding: The work of the first author was partially supported by the Science Challenge project TZ2016002, the National Natural Science Foundation of China grants 11771434, 91330202, and the National Center for Mathematics and Interdisciplinary Science, CAS. The work of the second author was partially supported by the National Natural Science Foundation of China grants 11871339, 11861131004, and 11571314. The work of the third author was supported by the Air Force Office of Scientific Research (AFOSR) DARPA EQUiPS program under award number FA9550-16-1-0054 (Computational Information Games), the Air Force Office of Scientific Research under award number FA9550-18-1-0271 (Games for Computation and Learning), and the Office of Naval Research under award N00014-18-1-2363 (Toward Scalable Universal Solvers for Linear Systems).

$\dagger$ LSEC, Academy of Mathematics and Systems Science, Chinese Academy of Sciences, No. 55, Zhongguancun Donglu, Beijing, 100190, and School of Mathematical Sciences, University of Chinese Academy of Sciences, Beijing, 100049 China (hhxie@lsec.cc.ac.cn).

${ }^{\ddagger}$ Corresponding author. School of Mathematical Sciences, Institute of Natural Sciences, and Ministry of Education Key Laboratory in Scientific and Engineering Computing, Shanghai Jiao Tong University, Shanghai, 200240 China (lzhang2012@sjtu.edu.cn).

$\S$ Computing and Mathematical Sciences, California Institute of Technology, Pasadena, CA 91125 (owhadi@caltech.edu). 
and nonlinear solver based methods have been pursued in the recent years. For example, the Implicitly Restarted Lanczos/Arnoldi Method (IRLM/IRAM) [49], the Preconditioned INVerse ITeration (PINVIT) method [19, 10, 24], the Locally Optimal Block Preconditioned Conjugate Gradient (LOBPCG) method [25, 28], and the Jacobi-Davidson-type techniques [7] have been developed. For those state-of-the-art eigensolvers, the efficient application of preconditioning [24] is often crucial for the faster convergence and the reduction of computation cost, especially for multiscale eigenproblems.

Recently, two-level $[57,37]$ and multilevel $[34,35,36,55,56]$ correction methods have been proposed to reduce the complexity of solving eigenpairs associated with low eigenvalues by first solving a coarse mesh/scale approximation, which can then be corrected by solving linear systems (corresponding to linearized eigenvalue problems) on a hierarchy of finer meshes/scales. Although the multilevel correction approach has been extended to multigrid methods for linear and nonlinear eigenvalue problems $[16,34,35,36,23,55,56]$, the regularity estimates required for linear complexity do not hold for PDEs with rough coefficients and a naive application of the correction approach to multiscale eigenvalue problems may converge very slowly. For twolevel methods [57] this lack of robustness can be alleviated by numerical homogenization techniques [37], e.g., the so-called Localized Orthogonal Decomposition (LOD) method. For multilevel methods, gamblets [41, 42, 44, 48, 43] (operator-adapted wavelets satisfying three desirable properties: scale orthogonality, well-conditioned multiresolution decomposition, and localization) provide a natural multiresolution decomposition ensuring robustness for multiscale eigenproblems. As described in [43, sect. 5.1.3], these three properties are analogous to those required of Wannier functions $[29,54]$, which can be characterized as linear combinations $\chi_{i}=\sum_{j} c_{i, j} v_{j}$ of eigenfunctions $v_{j}$ associated with eigenvalues $\lambda_{j}$ such that the size of $c_{i, j}$ is large for $\lambda_{j}$ close to $\lambda_{i}$ and small otherwise, and such that the resulting linear combinations $\chi_{i}$ are concentrated in space.

The aim of this paper is, therefore, to design a fast multilevel numerical method for multiscale eigenvalue problems (e.g., for PDEs that may have rough and highly oscillatory coefficients) associated with a bijective positive symmetric linear operator $\mathcal{L}$, by integrating the multilevel correction approach with the gamblet multiresolution decomposition. In this merger, the gamblet decomposition supplies a hierarchy of coarse (sub)spaces for the multilevel correction method. The overall computational cost is that of solving a sequence of linear problems over this hierarchy (using a gamblet based multigrid approach [41]). Recently, Hou et. al. [21] proposed computing the leftmost eigenpairs of a sparse symmetric positive matrix by combining the implicitly restarted Lanczos method with a gamblet-like multiresolution decomposition where local eigenfunctions are used as measurement functions. This paper shows that the gamblet multilevel decomposition (1) enhances the convergence rate of eigenvalue solvers by enabling (through a gamblet based multigrid method) the fast and robust convergence of inner iterations (linear solves) in the multilevel correction method, and (2) provides efficient preconditioners for state-of-the-art eigensolvers such as the LOBPCG method.

Outline. This paper is organized as follows: We summarize the gamblet decomposition, its properties, and the gamblet based multigrid method in section 2 (see $[41,42,44,48,43]$ for the detailed construction). We present the gamblet based multilevel method for multiscale eigenvalue problems and its rigorous analysis in section 3 . Our theoretical results are numerically illustrated in section 4 where the proposed method is compared with state-of-the-art eigensolvers (such as LOBPCG). 
Notation. The symbol $C$ denotes generic positive constant that may change from one line of an estimate to the next. $C$ will be independent from the eigenvalues (otherwise a subscript $\lambda$ will be added), and the dependencies of $C$ will normally be clear from the context or stated explicitly.

2. Gamblet decomposition and gamblet based multigrid method. Although multigrid methods $[11,20]$ have been highly successful in solving elliptic PDEs, their convergence rates can be severely affected by the lack of regularity of the PDE coefficients [53]. Although classical wavelet based methods [13, 17] enable a multiresolution decomposition of the solution space, their performance can also be affected by their lack of adaptation to the coefficients of the PDE. The introduction of gamblets in [41] addressed the problem of designing multigrid/multiresolution methods that are provably robust with respect to rough $\left(L^{\infty}\right)$ PDE coefficients.

Gamblets are derived from a game theoretic approach to numerical analysis [41, 42]. They are (1) elementary solutions of hierarchical information games associated with the process of computing with partial information and limited resources, (2) have a natural Bayesian interpretation under the mixed strategy emerging from the game theoretic formulation, and (3) induce a multiresolution decomposition of the solution space that is adapted to the numerical discretization of the underlying PDE. The (fast) gamblet transform has $\mathcal{O}\left(N \log ^{2 d+1} N\right)$ complexity for the first solve and $\mathcal{O}\left(N \log ^{d+1} N\right)$ for subsequent solves to achieve grid-size accuracy in $H^{1}$-norm for elliptic problems [43].

2.1. The abstract setting. We introduce the formulation of gamblets with an abstract setting since its application is not limited to scalar elliptic problems such as Examples 2.1 and 2.2. Let $(V,\|\cdot\|),\left(V^{*},\|\cdot\|_{*}\right)$, and $\left(V_{0},\|\cdot\|_{0}\right)$ be Hilbert spaces such that $V \subset V_{0} \subset V^{*}$ and such that the natural embedding $i: V_{0} \rightarrow V^{*}$ is compact and dense. Let $\left(V^{*},\|\cdot\|_{*}\right)$ be the dual of $(V,\|\cdot\|)$ using the dual pairing obtained from the Gelfand triple.

Let the operator $\mathcal{L}$ be a symmetric positive linear bijection mapping $V$ to $V^{*}$. Write $[\cdot, \cdot]$ for the duality pairing between $V^{*}$ and $V$ (derived from the Riesz duality between $V_{0}$ and itself) such that

$$
\|u\|^{2}=[\mathcal{L} u, u] \quad \text { for } u \in V .
$$

The corresponding inner product on $V$ is defined by

$$
\langle u, v\rangle:=[\mathcal{L} u, v] \quad \text { for } u, v \in V,
$$

and $\|\cdot\|_{*}$ is the corresponding dual-norm on $V^{*}$, i.e.,

$$
\|\phi\|_{*}=\sup _{v \in V, v \neq 0} \frac{[\phi, v]}{\|v\|} \quad \text { for } \phi \in V^{*} .
$$

Given $g \in V^{*}$, we will consider the solution $u$ of the variational problem

$$
\langle u, v\rangle=[g, v] \quad \text { for } v \in V .
$$

Example 2.1. Let $\Omega$ be a bounded open subset of $\mathbb{R}^{d}$ (of arbitrary dimension $d \in \mathbb{N}^{*}$ ) with uniformly Lipschitz boundary. Given $s \in \mathbb{N}$, let

$$
\mathcal{L}: \mathcal{H}_{0}^{s}(\Omega) \rightarrow \mathcal{H}^{-s}(\Omega)
$$


be a continuous linear bijection between $\mathcal{H}_{0}^{s}(\Omega)$ and $\mathcal{H}^{-s}(\Omega)$, where $\mathcal{H}_{0}^{s}(\Omega)$ is the Sobolev space of order $s$ with zero trace, and $\mathcal{H}^{-s}(\Omega)$ is the topological dual of $\mathcal{H}_{0}^{s}(\Omega)$ [1]. Assume $\mathcal{L}$ to be symmetric, positive, and local, i.e., $[\mathcal{L} u, v]=[u, \mathcal{L} v]$ and $[\mathcal{L} u, u] \geq$ 0 for $u, v \in \mathcal{H}_{0}^{s}(\Omega)$ and $[\mathcal{L} u, v]=0$ if $u, v$ have disjoint supports in $\Omega$. In this example $V, V^{*}$ and $V_{0}$ are $\mathcal{H}_{0}^{s}(\Omega), \mathcal{H}^{-s}(\Omega)$, and $L^{2}(\Omega)$ endowed with the norms $\|u\|^{2}=\int_{\Omega} u \mathcal{L} u$, $\|\phi\|_{*}^{2}=\int_{\Omega} \phi \mathcal{L}^{-1} \phi$, and $\|u\|_{0}=\|u\|_{L^{2}(\Omega)}$.

Example 2.2. Consider Example 2.1 with $s=1, \mathcal{L}=-\operatorname{div}(a(x) \nabla \cdot)$, and $a(x)$ is a symmetric, uniformly elliptic $d \times d$ matrix with entries in $L^{\infty}(\Omega)$ such that for all $x \in \Omega$ and $\ell \in \mathbb{R}^{d}$,

$$
\lambda_{\min }(a)|\ell|^{2} \leq \ell^{T} a(x) \ell \leq \lambda_{\max }(a)|\ell|^{2} .
$$

Note that

$$
\|v\|^{2}=\int_{\Omega}(\nabla v)^{T} a \nabla v \quad \text { for } v \in \mathcal{H}_{0}^{1}(\Omega)
$$

and the solution of (2.4) is the solution of the PDE

$$
\left\{\begin{array}{l}
-\operatorname{div}(a(x) \nabla u(x))=g(x) \quad x \in \Omega, \\
u=0 \text { on } \partial \Omega .
\end{array}\right.
$$

2.2. Gamblets. Here we give a brief reminder of the construction of gamblets. See Example 2.4 for a concrete example for scalar elliptic equation and section 4.1 for the numerical implementation, and also [41, 42, 44, 48, 43] for more details.

Measurement functions. Let $\mathcal{I}^{(1)}, \ldots, \mathcal{I}^{(q)}$ be a hierarchy of labels, and let $\phi_{i}^{(k)}$ be a hierarchy of nested elements of $V^{*}$ such that

$$
\phi_{i}^{(k)}=\sum_{j \in \mathcal{I}^{(k+1)}} \pi_{i, j}^{(k, k+1)} \phi_{j}^{(k+1)} \quad \text { for } k \in\{1, \ldots, q-1\} \text { and } i \in \mathcal{I}^{(k)}
$$

for some rank $\left|\mathcal{I}^{(k)}\right|, \mathcal{I}^{(k)} \times \mathcal{I}^{(k+1)}$ matrices $\pi^{(k, k+1)}$ and such that the $\left(\phi_{i}^{(k)}\right)_{i \in \mathcal{I}^{(k)}}$ are linearly independent and $\pi^{(k, k+1)} \pi^{(k+1, k)}=I_{\mathcal{I}^{(k)}}$ for $k \in\{1, \ldots, q-1\}$ (writing $I_{\mathcal{J}}$ for the $\mathcal{J} \times \mathcal{J}$ identity matrix and $\pi^{(k+1, k)}$ for $\left.\left(\pi^{(k, k+1)}\right)^{T}\right)$. Although not required in the general theory of gamblets [43] in this paper we assume that the $\phi_{i}^{(k)}$ are elements of $V_{0}$ and have uniformly well conditioned mass matrices in the sense that $C^{-1}|x|^{2} \leq\left\|\sum_{i} x_{i} \phi_{i}^{(k)}\right\|_{0}^{2} \leq C|x|^{2}$ (for all $x$ and $k$ ).

Operator adapted prewavelets. For $k \in\{1, \ldots, q\}$, let $\Theta^{(k)}$ be the symmetric positive definite matrix with entries $\Theta_{i, j}^{(k)}:=\left[\phi_{i}^{(k)}, \mathcal{L}^{-1} \phi_{j}^{(k)}\right]$, and (writing $\Theta^{(k),-1}$ for the inverse of $\left.\Theta^{(k)}\right)$ let

$$
\psi_{i}^{(k)}=\sum_{j \in \mathcal{I}^{(k)}} \Theta_{i, j}^{(k),-1} \phi_{j}^{(k)} \quad \text { for } i \in \mathcal{I}^{(k)} .
$$

The elements $\psi_{i}^{(k)}$ form a biorthogonal system with respect to the elements $\phi_{i}^{(k)}$, i.e., $\left[\phi_{j}^{(k)}, \psi_{i}^{(k)}\right]=\delta_{i, j}$ and

$$
u^{(k)}:=\sum_{i \in \mathcal{I}^{(k)}}\left[\phi_{i}^{(k)}, u\right] \psi_{i}^{(k)}
$$

Copyright (c) by SIAM. Unauthorized reproduction of this article is prohibited. 
is the $\langle\cdot, \cdot\rangle$ orthogonal projection of $u \in V$ onto

$$
\mathfrak{V}^{(k)}:=\operatorname{span}\left\{\psi_{i}^{(k)} \mid i \in \mathcal{I}^{(k)}\right\} .
$$

Furthermore, $A^{(k)}:=\Theta^{(k),-1}$ can be identified as the stiffness matrix of the $\psi_{i}^{(k)}$, i.e.,

$$
A_{i, j}^{(k)}=\left\langle\psi_{i}^{(k)}, \psi_{j}^{(k)}\right\rangle \quad \text { for } i, j \in \mathcal{I}^{(k)} .
$$

The $\psi_{i}^{(k)}$ are nested prewavelets in the sense that $\mathfrak{V}^{(k)} \subset \mathfrak{V}^{(k+1)}$ and

$$
\psi_{i}^{(k)}=\sum_{j \in \mathcal{I}^{(k+1)}} R_{i, j}^{(k, k+1)} \psi_{j}^{(k+1)},
$$

where $R^{(k, k+1)}=A^{(k)} \pi^{(k, k+1)} \Theta^{(k+1)}$ acts as an interpolation matrix.

Gamblets (operator adapted wavelets). Let $\left(\mathcal{J}^{(k)}\right)_{2 \leq k \leq q}$ be a hierarchy of labels such that (writing $\left|\mathcal{J}^{(k)}\right|$ for the cardinal of $\left.\mathcal{J}^{(k)}\right)\left|\mathcal{J}^{(k)}\right|=\left|\mathcal{I}^{(k)}\right|-\left|\mathcal{I}^{(k-1)}\right|$. For $k \in\{2, \ldots, q\}$, let $W^{(k)}$ be a $\mathcal{J}^{(k)} \times \mathcal{I}^{(k)}$ matrix such that (writing $W^{(k), T}$ for the transpose of $\left.W^{(k)}\right)$

$$
\operatorname{Ker}\left(\pi^{(k-1, k)}\right)=\operatorname{Im}\left(W^{(k), T}\right) \text { and } W^{(k)} W^{(k), T}=I_{\mathcal{J}^{(k)}} .
$$

Define

$$
\chi_{i}^{(k)}:=\sum_{j \in \mathcal{I}^{(k)}} W_{i, j}^{(k)} \psi_{j}^{(k)} \quad k \in\{2, \ldots, q\} \text { and } i \in \mathcal{J}^{(k)} .
$$

Then $u^{(k)}-u^{(k-1)}$ is the $\langle\cdot, \cdot\rangle$ orthogonal projection of $u \in V$ onto

$$
\mathfrak{W}^{(k)}:=\operatorname{span}\left\{\chi_{i}^{(k)} \mid i \in \mathcal{J}^{(k)}\right\} .
$$

We will also write $\mathcal{J}^{(1)}:=\mathcal{I}^{(1)}, \chi_{i}^{(1)}:=\psi_{i}^{(1)}, \mathfrak{W}^{(1)}:=\mathfrak{V}^{(1)}$. We call those operator adapted wavelets $\chi_{i}^{(k)}$, gamblets. Furthermore, $\mathfrak{W}^{(k)}$ is the $\langle\cdot, \cdot\rangle$-orthogonal complement of $\mathfrak{V}^{(k-1)}$ in $\mathfrak{V}^{(k)}$, i.e. $\mathfrak{V}^{(k)}=\mathfrak{V}^{(k-1)} \oplus \mathfrak{W}^{(k)}$,

$$
\mathfrak{V}^{(q)}=\mathfrak{V}^{(1)} \oplus \mathfrak{W}^{(2)} \oplus \cdots \oplus \mathfrak{W}^{(q)},
$$

and writing $\mathfrak{W}^{(q+1)}$ for the $\langle\cdot, \cdot\rangle$-orthogonal complement of $\mathfrak{V}^{(q)}$ in $V, u=u^{(1)}+$ $\left(u^{(2)}-u^{(1)}\right)+\cdots+\left(u^{(q+1)}-u^{(q)}\right)$ is the multiresolution decomposition of $u$ over $V=\mathfrak{V}^{(1)}+\mathfrak{W}^{(2)}+\cdots+\mathfrak{W}^{(q+1)}$, namely, the gamblet decomposition of $u$.

For $k \in\{2, \ldots, q\}, B^{(k)}=W^{(k)} A^{(k)} W^{(k), T}$ is the stiffness matrix of the $\chi_{i}^{(k)}$, i.e.,

$$
B_{i, j}^{(k)}=\left\langle\chi_{i}^{(k)}, \chi_{j}^{(k)}\right\rangle \quad \text { for } i, j \in \mathcal{J}^{(k)}
$$

and $B^{(1)}:=A^{(1)}$.

Quantitative estimates. Under general stability conditions on the $\phi_{i}^{(k)}[41,44,42$, $48,43]$ these operator adapted wavelets satisfy the quantitative estimates of Property 2.3 , we will first state those estimates and provide an example of their validity in the general setting of Example 2.1.

Property 2.3. The following properties are satisfied for some constant $C>0$ and $H \in(0,1)$.

Copyright (C) by SIAM. Unauthorized reproduction of this article is prohibited. 
1. Approximation:

$$
\left\|u-u^{(k)}\right\|_{0} \leq C H^{k}\left\|u-u^{(k)}\right\| \text { for } u \in V,
$$

and

$$
\left\|u-u^{(k)}\right\| \leq C H^{k}\|\mathcal{L} u\|_{0} \text { for } u \in \mathcal{L}^{-1} V_{0} .
$$

2. Uniform bounded condition number: Writing $\operatorname{Cond}(B)$ for the condition number of a matrix $B$ we have for $k \in\{1, \ldots, q\}$,

$$
C^{-1} H^{-2(k-1)} I_{\mathcal{J}^{(k)}} \leq B^{(k)} \leq C H^{-2 k} I_{\mathcal{J}^{(k)}} \text { and } \operatorname{Cond}\left(B^{(k)}\right) \leq C H^{-2}
$$

and

$$
C^{-1} I_{\mathcal{I}^{(k)}} \leq A^{(k)} \leq C H^{-2 k} I_{\mathcal{I}^{(k)}} .
$$

3. Near linear complexity: The wavelets $\psi_{i}^{(k)}, \chi_{i}^{(k)}$ and stiffness matrices $A^{(k)}, B^{(k)}$ can be computed to precision $\varepsilon$ (in $\|\cdot\|$-energy norm for elements of $V$ and in Frobenius norm for matrices) in $\mathrm{O}\left(N\right.$ polylog $\left.\frac{N}{\varepsilon}\right)$ complexity.

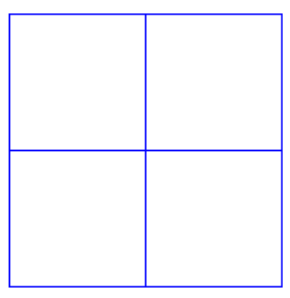

$\Omega$
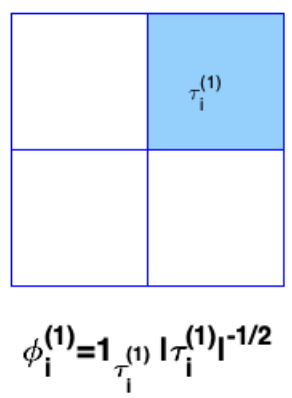

\begin{tabular}{|l|l|l|l|}
\hline 0 & 0 & $1 / 2$ & $1 / 2$ \\
\hline 0 & 0 & $1 / 2$ & $1 / 2$ \\
\hline 0 & 0 & 0 & 0 \\
\hline 0 & 0 & 0 & 0 \\
\hline
\end{tabular}

$\pi_{i, \text {. }}^{(1,2)}$

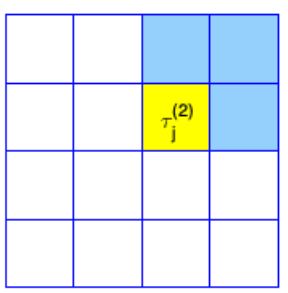

$\phi_{\mathbf{j}}^{(2)}$

\begin{tabular}{|l|l|l|l|l|l|l|l|}
\hline 0 & 0 & 0 & 0 & 0 & 0 & 0 & 0 \\
\hline 0 & 0 & 0 & 0 & 0 & 0 & 0 & 0 \\
\hline 0 & 0 & 0 & 0 & $1 / 2$ & $1 / 2$ & 0 & 0 \\
\hline 0 & 0 & 0 & 0 & $1 / 2$ & $1 / 2$ & 0 & 0 \\
\hline 0 & 0 & 0 & 0 & 0 & 0 & 0 & 0 \\
\hline 0 & 0 & 0 & 0 & 0 & 0 & 0 & 0 \\
\hline 0 & 0 & 0 & 0 & 0 & 0 & 0 & 0 \\
\hline 0 & 0 & 0 & 0 & 0 & 0 & 0 & 0 \\
\hline
\end{tabular}

$\pi_{\mathrm{i}, \cdot}^{(2,3)}$

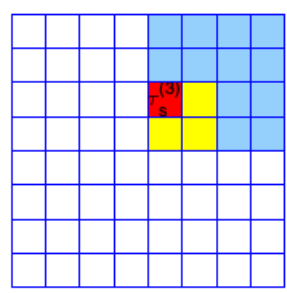

$\phi_{\mathbf{s}}^{(3)}$

FIG. 1. Nested partition of $\Omega=(0,1)^{2}$ such that the kth level corresponds to a uniform partition of $\Omega$ into $2^{-k} \times 2^{-k}$ squares. The top row shows the entries of $\pi_{i, \cdot}^{(1,2)}$ and $\pi_{j, \cdot}^{(2,3)}$. The bottom row shows the support of $\phi_{i}^{(1)}, \phi_{j}^{(2)}$, and $\phi_{s}^{(3)}$. Note that $j^{(1)}=s^{(1)}=i$ and $s^{(2)}=j$.

Example 2.4. Consider Example 2.1. Let $\mathcal{I}^{(q)}$ be the finite set of $q$-tuples of the form $i=\left(i_{1}, \ldots, i_{q}\right)$. For $1 \leq k<q$ and a $r$-tuple of the form $i=\left(i_{1}, \ldots, i_{q}\right)$, write

Copyright (c) by SIAM. Unauthorized reproduction of this article is prohibited. 
$i^{(k)}:=\left(i_{1}, \ldots, i_{k}\right)$. For $1 \leq k \leq q$ and $i=\left(i_{1}, \ldots, i_{q}\right) \in \mathcal{I}^{(q)}$, write $\mathcal{I}^{(k)}:=\left\{i^{(k)}: i \in\right.$ $\left.\mathcal{I}^{(q)}\right\}$. Let $\delta, h \in(0,1)$. Let $\left(\tau_{i}^{(k)}\right)_{i \in \mathcal{I}^{(k)}}$ be uniformly Lipschitz convex sets forming a nested partition of $\Omega$, i.e., such that $\Omega=\cup_{i \in \mathcal{I}^{(k)}} \tau_{i}^{(k)}, k \in\{1, \ldots, q\}$ is a disjoint union except for the boundaries, and $\tau_{i}^{(k)}=\cup_{j \in \mathcal{I}^{(k+1)}: j^{(k)}=i} \tau_{j}^{(k+1)}, k \in\{1, \ldots, q-1\}$. Assume that each $\tau_{i}^{(k)}$ contains a ball of center $x_{i}^{(k)}$ and radius $\delta h^{k}$, and is contained in the ball of center $x_{i}^{(k)}$ and radius $\delta^{-1} h^{k}$. Writing $\left|\tau_{i}^{(k)}\right|$ for the volume of $\tau_{i}^{(k)}$, take

$$
\phi_{i}^{(k)}:=1_{\tau_{i}^{(k)}}\left|\tau_{i}^{(k)}\right|^{-\frac{1}{2}} .
$$

The nesting relation (2.9) is then satisfied with $\pi_{i, j}^{(k, k+1)}:=\left|\tau_{j}^{(k+1)}\right|^{\frac{1}{2}}\left|\tau_{i}^{(k)}\right|^{-\frac{1}{2}}$ for $j^{(k)}=$ $i$ and $\pi_{i, j}^{(k, k+1)}:=0$, otherwise. Observe also that $\left\|\sum_{i} x_{i} \phi_{i}^{(k)}\right\|_{L^{2}(\Omega)}^{2}=|x|^{2}$. For $i:=\left(i_{1}, \ldots, i_{k+1}\right) \in \mathcal{I}^{(k+1)}$ write $i^{(k)}:=\left(i_{1}, \ldots, i_{k}\right) \in \mathcal{I}^{(k)}$ and note that $\pi^{(k, k+1)}$ is cellular in the sense that $\pi_{i, j}^{(k, k+1)}=0$ for $j^{(k)} \neq i$. Choose $\left(\mathcal{J}^{(k)}\right)_{2 \leq k \leq q}$ to be a finite set of $k$-tuples of the form $j=\left(j_{1}, \ldots, j_{k}\right)$ such that $j^{(k-1)}:=\left(j_{1}, \ldots, j_{k-1}\right) \in \mathcal{I}^{(k-1)}$ and $\left|\mathcal{J}^{(k)}\right|=\left|\mathcal{I}^{(k)}\right|-\left|\mathcal{I}^{(k-1)}\right|$. See Figure 1 for an illustration. Choose $W^{(k)}$ as in (2.15) and cellular in the sense that $W_{i, j}^{(k)}=0$ for $i^{(k-1)} \neq j^{(k-1)}$ (see $[41,42,43,44]$ for examples). Equation (2.18) then corresponds to a multiresolution decomposition of $\mathcal{H}_{0}^{s}(\Omega)$ that is adapted to the operator $\mathcal{L}$.

We have the following theorem $[42,43,48]$.

TheOREm 2.5. The properties in Property 2.3 are satisfied for Examples 2.1 and 2.4 with $H=h^{s}$ and a constant $C$ depending only on $\delta, \Omega, d, s$,

$$
\|\mathcal{L}\|:=\sup _{u \in \mathcal{H}_{0}^{s}(\Omega)} \frac{\|\mathcal{L} u\|_{\mathcal{H}^{-s}(\Omega)}}{\|u\|_{\mathcal{H}_{0}^{s}(\Omega)}} \quad \text { and } \quad\left\|\mathcal{L}^{-1}\right\|:=\sup _{u \in \mathcal{H}_{0}^{s}(\Omega)} \frac{\|u\|_{\mathcal{H}_{0}^{s}(\Omega)}}{\|\mathcal{L} u\|_{\mathcal{H}^{-s}(\Omega)}} .
$$

Furthermore, the wavelets $\chi_{i}^{(k)}$ and $\psi_{i}^{(k)}$ are exponentially localized, i.e.,

$\left\|\psi_{i}^{(k)}\right\|_{\mathcal{H}^{s}\left(\Omega \backslash B\left(x_{i}^{(k)}, n h^{k}\right)\right)} \leq C h^{-s k} e^{-n / C}$ and $\left\|\chi_{i}^{(k)}\right\|_{\mathcal{H}^{s}\left(\Omega \backslash B\left(x_{i}^{(k)}, n h^{k-1}\right)\right)} \leq C h^{-s k} e^{-n / C}$,

and the wavelets $\psi_{i}^{(k)}, \chi_{i}^{(k)}$ and stiffness matrices $A^{(k)}, B^{(k)}$ can be computed to precision $\varepsilon$ (in $\|\cdot\|$-energy norm for elements of $V$ and in Frobenius norm for matrices) in $\mathrm{O}\left(N \log ^{2 d+1} \frac{N}{\varepsilon}\right)$ complexity [43].

Remark 2.6. Rigorous exponential decay/localization results such as (2.26) have been pioneered in [38] for the LOD basis functions. Although gamblets are derived from a different perspective (namely, a game theoretic approach), from the numerical point of view, gamblets can be seen as a multilevel generalization of optimal recovery splines [39] and of numerical homogenization basis functions such as RPS (rough polyharmonic splines) [45] and variational multiscale/LOD basis functions [22, 38].

Remark 2.7. For Examples 2.1 and 2.4, the wavelets $\psi_{i}^{(k)}, \chi_{i}^{(k)}$ and stiffness matrices $A^{(k)}, B^{(k)}$ can also be computed in $\mathrm{O}\left(N \log ^{2 d} \frac{N}{\varepsilon}\right)$ complexity using the incomplete Cholesky factorization approach of [48].

Discrete case. From now on we will consider the situation where $V$ is finitedimensional and $\mathfrak{V}^{(q)}=V$. In the setting of Example 2.1 we will identify $V$ with the linear space spanned by the finite-elements $\tilde{\psi}_{i}$ (e.g., piecewise linear or bilinear tent functions on a fine mesh/grid in the setting of Example 2.2) used to discretize the 
operator $\mathcal{L}$, use $\mathcal{I}^{(q)}$ to label the elements $\tilde{\psi}_{i}$ and set $\psi_{i}^{(q)}=\tilde{\psi}_{i}^{(q)}$ for $i \in \mathcal{I}^{(q)}$. The gamblet transform [41, 42, 43] is then summarized in Algorithm 2.1 and we have the decompsoition

$$
V=\mathfrak{W}^{(1)} \oplus \mathfrak{W}^{(2)} \oplus \cdots \oplus \mathfrak{W}^{(q)} .
$$

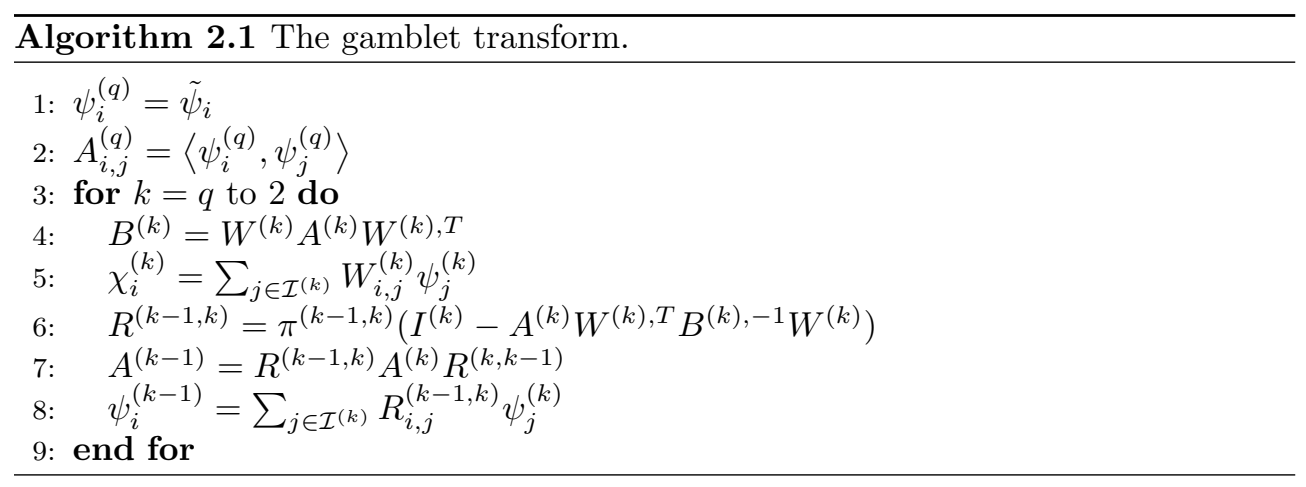

Fast gamblet transform. The acceleration of Algorithm 2.1 to $\mathrm{O}\left(N \log ^{2 d+1} \frac{N}{\varepsilon}\right)$ complexity is based on the truncation and localization of the computation of the interpolation matrices $R^{(k, k+1)}$ that is enabled by the exponential decay of gamblets and the uniform bound on $\operatorname{Cond}\left(B^{(k)}\right)$. In the setting of Examples 2.1 and 2.4, this acceleration is equivalent to localizing the computation of each gamblet $\psi_{i}^{(k)}$ to a subdomain centered on $\tau_{i}^{(k)}$ and of diameter $\mathcal{O}\left(H^{k} \log \frac{1}{H^{k}}\right)$. We refer to [41, 42, 43] for a detailed description of this acceleration.

Higher order problems. Although the local linear elliptic operators of Example 2.1 are used as prototypical examples, the proposed theory and algorithms is presented in the abstract setting of linear operators on Hilbert spaces to not only emphasize the generality of the proposed method (which could also be applied to Graph Laplacians with well behaved gamblets) but also to clarify/simplify its application to higher order eigenvalue problems. For such applications the method is directly applied to the SPD matrix representation $A$ of the discretized operator as described in [43, Chap. 21]. The identification of level $q$ gamblets $\psi_{i}^{(q)}$ in step 1 of Algorithm 2.1 with the finite elements $\tilde{\psi}_{i}$ used to discretize the operator is, when the gamblet transform is applied to the SPD matrix $A$, equivalent to the identification of level $q$ gamblets $\psi_{i}^{(q)}$ with the unit vectors of $\mathbb{R}^{N}$. The nesting matrices $\pi^{(k-1, k)}$ remain those associated with the Haar prewavelets of Example 2.4 (the algorithm does not require the explicit input of measurement functions, only those nesting matrices are used as inputs and they remain unchanged). Of course, fine scale finite elements $\tilde{\psi}_{i}$ (used to discretize the operator) have to be of sufficient accuracy for the approximation of the required eigenpairs (see $[12,60]$ and references therein for further discussion of the discretization issue).

2.3. Gamblet based multigrid method. The gamblet decomposition enables the construction of efficient multigrid solvers and preconditioners. Suppose we have computed the decomposition (2.27), the stiffness matrices $A^{(k)}$ and interpolation matrices $R^{(k-1, k)}$ in Algorithm 2.1, or more precisely their numerical approximations using the fast gamblet transform [41, 42, 48, 43], to a degree that is sufficient to obtain grid-size accuracy in the resolution of the discretization of (2.4). We will write 
$R^{(k, k-1)}:=\left(R^{(k-1, k)}\right)^{T}$ for the restriction matrix associated with the interpolation matrix $R^{(k-1, k)}$.

For $g^{(k)} \in \mathbb{R}^{\mathcal{I}^{(k)}}$ consider the linear system

$$
A^{(k)} z=g^{(k)} \text {. }
$$

Algorithm 2.2 provides a multigrid approximation $\mathrm{MG}\left(k, z_{0}, g^{(k)}\right)$ of the solution $z$ of (2.28) based on an initial guess $z_{0}$ and a number of iterations $k$. In that algorithm, $m_{1}$ and $m_{2}$ are nonnegative integers (and $p=1$ or 2. $p=1$ corresponds to a $\mathcal{V}$-cycle method and $p=2$ corresponds to a $\mathcal{W}$-cycle method). $\Lambda^{(k)}$ is an upper bound for the spectral radius of $A^{(k)}$. Under Property 2.3 we take $\Lambda^{(k)}=C H^{-2 k}$ where $C$ and $H$ are the constants appearing in the bound $A^{(k)} \leq C H^{-2 k} I_{\mathcal{I}^{(k)}}$.

Remark 2.8. We use the simple Richardson iteration in the smoothing step of Algorithm 2.2. In practice, Gauss-Seidel and CG can also be used as a smoother.

Remark 2.9. The number of operations required in the $k$ th level iteration defined by Algorithm 2.2 is $\mathcal{O}\left(N_{k}\left(\log \frac{N_{k}}{\varepsilon}\right)^{2 d+1}\right)$, where $N_{k}:=\operatorname{dim}\left(\mathfrak{V}^{(k)}\right)$.

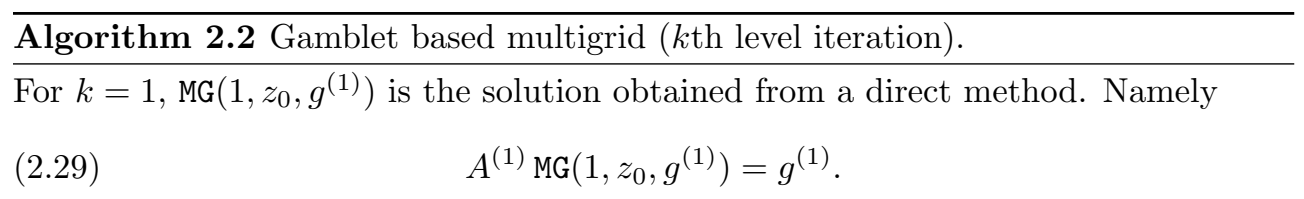

For $k>1, \operatorname{MG}\left(k, z_{0}, g^{(k)}\right)$ is obtained recursively in three steps.

1. Presmoothing: For $1 \leq \ell \leq m_{1}$, let

$$
z_{\ell}=z_{\ell-1}+\frac{1}{\Lambda^{(k)}}\left(g^{(k)}-A^{(k)} z_{\ell-1}\right) .
$$

2. Error correction: Let $g^{(k-1)}:=R^{(k-1, k)}\left(g^{(k)}-A^{(k)} z_{0}\right)$ and $q_{0}^{(k-1)}=0$. For $1 \leq i \leq p$, let

$$
q_{i}^{(k-1)}=\operatorname{MG}\left(k-1, q_{i-1}^{(k-1)}, g^{(k-1)}\right) .
$$

Then $z_{m_{1}+1}:=z_{m_{1}}+R^{(k, k-1)} q_{p}^{(k-1)}$.

3. Postsmoothing: For $m_{1}+2 \leq \ell \leq m_{1}+m_{2}+1$, let

$$
z_{\ell}=z_{\ell-1}+\frac{1}{\Lambda^{(k)}}\left(g^{(k)}-A^{(k)} z_{\ell-1}\right) .
$$

Then the output of the $k$ th level iteration is

$$
\operatorname{MG}\left(k, z_{0}, g^{(k)}\right):=z_{m_{1}+m_{2}+1} .
$$

Items 1 and 2 of Property 2.3 (i.e., the bounds on approximation errors and condition numbers) imply the following result of $\mathcal{V}$-cycle convergence.

TheOREM 2.10 (convergence of the $k$ th level iteration). Let $m_{1}=m_{2}=m / 2$, and $k$ be the level number of Algorithm 2.2, and $p=1$. For any $0<\theta<1$, there exists $m$ independent from $k$ such that

$$
\left\|z-M G\left(k, z_{0}, g\right)\right\|_{A^{(k)}} \leq \theta\left\|z-z_{0}\right\|_{A^{(k)}} .
$$

Copyright $@$ by SIAM. Unauthorized reproduction of this article is prohibited. 
Proof. Theorem 2.10 follows from the following smoothing and approximation properties introduced in [40, sect. 3.3.7].

Smoothing property: The iteration matrix on every grid level can be written as $S^{(k)}=I-M^{(k),-1} A^{(k)}$, where $M^{(k)}$ is symmetric and satisfies

$$
M^{(k)} \geq A^{(k)} \text {. }
$$

Approximation property: It holds true that

$$
\left\|A^{(k),-1}-R^{(k, k-1)} A^{(k-1),-1} R^{(k-1, k)}\right\|_{2} \leq C\left\|M^{(k)}\right\|_{2}^{-1} .
$$

Taking $M^{(k)}=A^{(k)} I^{(k)}=C H^{-2 k}$ in Algorithm 2.2 implies the smoothing property (2.35) by (2.23) in Property 2.3. There are two approaches to proving the approximation property (2.36). The first one would be to adapt the classical approach as presented in [40, p. 130] (in that approach (2.36) is implied by (2.20) and (2.21) and it requires the mass matrix of the gamblets to be well conditioned which follows from [58, Thm. 6.3]). Here we present a second approach. First, observe that $D:=A^{(k),-1}-R^{(k, k-1)} A^{(k-1),-1} R^{(k-1, k)}$ is symmetric and positive. Indeed, using $R^{(k-1, k)}=A^{(k-1)} \pi^{(k-1, k)} A^{(k),-1}$, we have for $z=A^{(k)} y, z^{T} D z=y^{T} A^{(k)} y-$ $y^{T} \pi^{(k, k-1)} A^{(k-1)} \pi^{(k-1, k)} y=\left\|u^{(k)}-u^{(k-1)}\right\|^{2}$ with $u^{(k)}=\sum_{i \in \mathcal{I}^{(k)}} y_{i} \psi_{i}^{(k)}$ (see [43, Prop. 13.30] for details). Therefore, $\|D\|_{2}=\sup _{|x|=1} x^{T} D x$. Now take $g=\sum_{i} x_{i} \phi_{i}^{(k)}$ and $u=\mathcal{L}^{-1} g$. Then $x^{T} A^{(k),-1} x=\|u\|^{2}$ and $x^{T} R^{(k, k-1)} A^{(k-1),-1} R^{(k-1, k)} x=$ $\left\|u^{(k-1)}\right\|^{2}$. Therefore $x^{T} D x=\|u\|^{2}-\left\|u^{(k-1)}\right\|^{2}=\left\|u-u^{(k-1)}\right\|^{2}$. Using (2.21) in Property 2.3 we have $\left\|u-u^{(k-1)}\right\|^{2} \leq C H^{2(k-1)}\|g\|_{0}^{2}$. Using $\|g\|_{0}^{2} \leq C|x|^{2}$ we deduce that $\|D\|_{2} \leq C H^{2(k-1)}$ which implies the result for $M^{(k)}=A^{(k)} I^{(k)}=C H^{-2 k}$ (a factor $H^{-2}$ is absorbed into $C$ ).

We conclude the proof of (2.34) by applying [40, Theorem 3.9], and taking $\theta \geq$ $\frac{C}{C+m}$, where $C$ is the constant in (2.36).

Remark 2.11. The LOD [38] based Schwarz subspace decomposition/correction method of $[31,30]$ also leads to a robust two-level preconditioning method for PDEs with rough coefficients. From a multigrid perspective, a multilevel version of [31, 30] would be closer to a domain decomposition/additive multigrid method compared to the proposed gamblet multigrid, which is a variant of the multiplicative multigrid method.

3. Gamblet subspace correction method for eigenvalue problem. We will now describe the gamblet based multilevel correction method. Consider the abstract setting of section 2.1 and write $\langle\cdot, \cdot\rangle_{0}$ for the scalar product associated with the norm $\|\cdot\|_{0}$ placed on $V_{0}$. Since $[\cdot, \cdot]$ is the dual product between $V^{*}$ and $V$ induced by the Gelfand triple $V \subset V_{0} \subset V^{*}$ we will also write $[u, v]:=\langle u, v\rangle_{0}$ for $u, v \in V_{0}$.

Consider the eigenvalue problem: Find $(\lambda, v) \in \mathbb{R} \times V$ such that $\langle v, v\rangle=1$, and

$$
\langle v, w\rangle=\lambda[v, w] \quad \forall w \in V .
$$

The compact embedding property implies that the eigenvalue problem (3.1) has a sequence of eigenvalues $\left\{\lambda_{j}\right\}$ (see $[6,15]$ ):

$$
0<\lambda_{1} \leq \lambda_{2} \leq \cdots \leq \lambda_{\ell} \leq \cdots, \quad \lim _{\ell \rightarrow \infty} \lambda_{\ell}=\infty,
$$

with associated eigenfunctions

$$
v_{1}, v_{2}, \ldots, v_{\ell}, \ldots
$$


where $\left\langle v_{i}, v_{j}\right\rangle=\delta_{i, j}\left(\delta_{i, j}\right.$ denotes the Kronecker function). In the sequence $\left\{\lambda_{j}\right\}$, the $\lambda_{j}$ 's are repeated according to their geometric multiplicity. For our analysis, recall the following definition for the smallest eigenvalue (see $[6,15])$ :

$$
\lambda_{1}=\min _{0 \neq w \in V} \frac{\langle w, w\rangle}{[w, w]} .
$$

Define the subspace approximation problem for eigenvalue problem (3.1) on $\mathfrak{V}^{(k)}$ as follows: Find $\left(\bar{\lambda}^{(k)}, \bar{v}^{(k)}\right) \in \mathbb{R} \times \mathfrak{V}^{(k)}$ such that $\left\langle\bar{v}^{(k)}, \bar{v}^{(k)}\right\rangle=1$ and

$$
\left\langle\bar{v}^{(k)}, w\right\rangle=\bar{\lambda}^{(k)}\left[\bar{v}^{(k)}, w\right] \quad \forall w \in \mathfrak{V}^{(k)} .
$$

From $[5,6,15]$, the discrete eigenvalue problem (3.3) has eigenvalues

$$
0<\bar{\lambda}_{1}^{(k)} \leq \bar{\lambda}_{2}^{(k)} \leq \cdots \leq \bar{\lambda}_{j}^{(k)} \leq \cdots \leq \bar{\lambda}_{N_{k}}^{(k)}
$$

and corresponding eigenfunctions

$$
\bar{v}_{1}^{(k)}, \bar{v}_{2}^{(k)}, \ldots, \bar{v}_{j}^{(k)} \ldots, \bar{v}_{N_{k}}^{(k)},
$$

where $\left\langle\bar{v}_{i}^{(k)}, \bar{v}_{j}^{(k)}\right\rangle=\delta_{i, j}, 1 \leq i, j \leq N_{k}$, and $N_{k}:=\operatorname{dim}\left(\mathfrak{V}^{(k)}\right)$.

From the min-max principle $[5,6]$, we have the following upper bound result:

$$
\lambda_{i} \leq \bar{\lambda}_{i}^{(k)}, \quad 1 \leq i \leq N_{k}
$$

Define

$$
\eta\left(\mathfrak{V}^{(k)}\right)=\sup _{f \in V_{0},\|f\|_{0}=1} \inf _{w \in \mathfrak{V}^{(k)}}\left\|\mathcal{L}^{-1} f-w\right\| .
$$

Let $M\left(\lambda_{i}\right)$ denote the eigenspace corresponding to the eigenvalue $\lambda_{i}$, namely,

$$
M\left(\lambda_{i}\right):=\left\{v \in V \mid\langle v, w\rangle=\lambda_{i}[v, w] \quad \forall w \in V\right\},
$$

and define

$$
\delta_{k}\left(\lambda_{i}\right)=\sup _{v \in M\left(\lambda_{i}\right),\|v\|=1} \inf _{w \in \mathfrak{V}(k)}\|v-w\| .
$$

Proposition 3.1. Property 2.3 implies

$$
\eta\left(\mathfrak{V}^{(k)}\right) \leq C H^{k}, \quad \delta_{k}\left(\lambda_{i}\right) \leq C \sqrt{\lambda_{i}} H^{k}, \quad \text { and } \quad \delta_{k}\left(\lambda_{i}\right) \leq \sqrt{\lambda_{i}} \eta\left(\mathfrak{V}^{(k)}\right),
$$

for $k \in\{1, \ldots, q\}$ where $C$ and $H$ are the constants appearing in Property 2.3.

In order to provide the error estimate for the numerical scheme (3.3), we define the corresponding projection operator $\mathcal{P}_{k}$ as follows:

$$
\left\langle\mathcal{P}_{k} u, w\right\rangle=\langle u, w\rangle \quad \forall w \in \mathfrak{V}^{(k)} .
$$

It is obvious that

$$
\left\|u-\mathcal{P}_{k} u\right\|=\inf _{w \in \mathfrak{V}^{(k)}}\|u-w\| .
$$

The following Rayleigh quotient expansion of the eigenvalue error is a useful tool to obtain error estimates for eigenvalue approximations.

Copyright (c) by SIAM. Unauthorized reproduction of this article is prohibited. 
Lemma 3.2 (see [5]). Assume $(\lambda, v)$ is an eigenpair for the eigenvalue problem (3.1). Then for any $w \in V \backslash\{0\}$, the following expansion holds:

$$
\frac{\langle w, w\rangle}{[w, w]}-\lambda=\frac{\langle w-u, w-u\rangle}{[w, w]}-\lambda \frac{[w-u, w-u]}{[w, w]} \quad \forall u \in M(\lambda) .
$$

For simplicity we will from now on restrict the presentation to the identification of a simple eigenpair $(\lambda, v)$ (the numerical method and results can naturally be extended to multiple eigenpairs). Let $E: V \rightarrow M\left(\lambda_{i}\right)$ be the spectral projection operator [5] defined by

$$
E=\frac{1}{2 \pi \mathrm{i}} \int_{\Gamma}(z-\mathcal{L})^{-1} d z
$$

where $\Gamma$ is a Jordan curve in $\mathbb{C}$ enclosing the desired eigenvalue $\lambda_{i}$ and no other eigenvalues.

We introduce the following lemma from [50] before stating error estimates of the subspace projection method.

Lemma 3.3 (see [50, Lemma 6.4]). For any exact eigenpair $(\lambda, v)$ of (3.1), the following equality holds:

$$
\left(\bar{\lambda}_{j}^{(k)}-\lambda\right)\left[\mathcal{P}_{k} v, \bar{v}_{j}^{(k)}\right]=\lambda\left[v-\mathcal{P}_{k} v, \bar{v}_{j}^{(k)}\right], \quad j=1, \ldots, N_{k} .
$$

The following lemma gives the error estimates for the gamblet subspace approximation, which is a direct application of the subspace approximation theory for eigenvalue problems; see [5, Lemma 3.6, Theorem 4.4] and [15].

Lemma 3.4. Let $(\lambda, v)$ denote an exact eigenpair of the eigenvalue problem (3.1). Assume the eigenpair approximation $\left(\bar{\lambda}_{i}^{(k)}, \bar{v}_{i}^{(k)}\right)$ has the property that $\bar{\mu}_{i}^{(k)}=1 / \bar{\lambda}_{i}^{(k)}$ is closest to $\mu=1 / \lambda$. The corresponding spectral projection $E_{i, k}: V \mapsto \operatorname{span}\left\{\bar{v}_{i}^{(k)}\right\}$ is defined as follows:

$$
\left\langle E_{i, k} w, \bar{v}_{i}^{(k)}\right\rangle=\left\langle w, \bar{v}_{i}^{(k)}\right\rangle \quad \forall w \in V .
$$

The eigenpair approximations $\left(\bar{\lambda}_{i}^{(k)}, \bar{v}_{i}^{(k)}\right)\left(i=1,2, \ldots, N_{k}\right)$ have the following error estimates:

$$
\begin{aligned}
\left\|E_{i, k} v-v\right\| & \leq \sqrt{1+\frac{1}{\lambda_{1} \delta_{\lambda}^{(k), 2}} \eta^{2}\left(\mathfrak{V}^{(k)}\right)} \delta_{k}\left(\lambda_{i}\right), \\
\left\|E_{i, k} v-v\right\|_{0} & \leq\left(1+\frac{1}{\lambda_{1} \delta_{\lambda}^{(k)}}\right) \eta\left(\mathfrak{V}^{(k)}\right)\left\|E_{i, k} v-v\right\|,
\end{aligned}
$$

where $\delta_{\lambda}^{(k)}$ is defined as follows:

$$
\delta_{\lambda}^{(k)}:=\min _{j \neq i}\left|\frac{1}{\bar{\lambda}_{j}^{(k)}}-\frac{1}{\lambda}\right|
$$

and $\delta_{\lambda}^{(k), 2}=\left(\delta_{\lambda}^{(k)}\right)^{2}$

Proof. Following a classical duality argument found in finite element method, we have

$$
\left\|\left(I-\mathcal{P}_{k}\right) u\right\|_{0}=\sup _{\|g\|_{0}=1}\left[\left(I-\mathcal{P}_{k}\right) u, g\right]=\sup _{\|g\|_{0}=1}\left\langle\left(I-\mathcal{P}_{k}\right) u, \mathcal{L}^{-1} g\right\rangle
$$

Copyright (C) by SIAM. Unauthorized reproduction of this article is prohibited. 


$$
=\sup _{\|g\|_{0}=1}\left\langle\left(I-\mathcal{P}_{k}\right) u,\left(I-\mathcal{P}_{k}\right) \mathcal{L}^{-1} g\right\rangle \leq \eta\left(\mathfrak{V}^{(k)}\right)\left\|\left(I-\mathcal{P}_{k}\right) u\right\| .
$$

Since $\left(I-E_{i, k}\right) \mathcal{P}_{k} v \in \mathfrak{V}^{(k)}$ and $\left\langle\left(I-E_{i, k}\right) \mathcal{P}_{k} v, \bar{v}_{i}^{(k)}\right\rangle=0$, the following orthogonal expansion holds:

$$
\left(I-E_{i, k}\right) \mathcal{P}_{k} v=\sum_{j \neq i} \alpha_{j} \bar{v}_{j}^{(k)}
$$

where $\alpha_{j}=\left\langle\mathcal{P}_{k} v, \bar{v}_{j}^{(k)}\right\rangle$. From Lemma 3.3, we have

$$
\begin{aligned}
\alpha_{j} & =\left\langle\mathcal{P}_{k} v, \bar{v}_{j}^{(k)}\right\rangle=\bar{\lambda}_{j}^{(k)}\left[\mathcal{P}_{k} v, \bar{v}_{j}^{(k)}\right]=\frac{\bar{\lambda}_{j}^{(k)} \lambda}{\bar{\lambda}_{j}^{(k)}-\lambda}\left[v-\mathcal{P}_{k} v, \bar{v}_{j}^{(k)}\right] \\
& =\frac{1}{\mu-\bar{\mu}_{j}^{(k)}}\left[v-\mathcal{P}_{k} v, \bar{v}_{j}^{(k)}\right]
\end{aligned}
$$

where $\mu=1 / \lambda$ and $\bar{\mu}_{j}^{(k)}=1 / \bar{\lambda}_{j}^{(k)}$.

From the property of eigenvectors $\bar{v}_{1}^{(k)}, \ldots, \bar{v}_{m}^{(k)}$, the following identities hold:

$$
1=\left\langle\bar{v}_{j}^{(k)}, \bar{v}_{j}^{(k)}\right\rangle=\bar{\lambda}_{j}^{(k)}\left[\bar{v}_{j}^{(k)}, \bar{v}_{j}^{(k)}\right]=\bar{\lambda}_{j}^{(k)}\left\|\bar{v}_{j}^{(k)}\right\|_{0}^{2},
$$

which leads to the following property:

$$
\left\|\bar{v}_{j}^{(k)}\right\|_{0}^{2}=\frac{1}{\bar{\lambda}_{j}^{(k)}}=\bar{\mu}_{j}^{(k)}
$$

From (3.3) and definitions of eigenvectors $\bar{v}_{1}^{(k)}, \ldots, \bar{u}_{m}^{(k)}$, we have the following equalities:

$$
\left\langle\bar{v}_{j}^{(k)}, \bar{v}_{i}^{(k)}\right\rangle=\delta_{i j}, \quad\left[\frac{\bar{v}_{j}^{(k)}}{\left\|\bar{v}_{j}^{(k)}\right\|_{0}}, \frac{\bar{v}_{i}^{(k)}}{\left\|\bar{v}_{i}^{(k)}\right\|_{0}}\right]=\delta_{i j}, \quad 1 \leq i, j \leq N_{k} .
$$

Combining (3.16), (3.17), (3.18), and (3.19), the following estimates hold:

$$
\begin{aligned}
& \left\|\left(I-E_{i, k}\right) \mathcal{P}_{k} v\right\|^{2}=\left\|\sum_{j \neq i} \alpha_{j} \bar{v}_{j}^{(k)}\right\|^{2}=\sum_{j \neq i} \alpha_{j}^{2}=\sum_{j \neq i}\left(\frac{1}{\mu-\bar{\mu}_{j}^{(k)}}\right)^{2}\left|\left[v-\mathcal{P}_{k} v, \bar{v}_{j}^{(k)}\right]\right|^{2} \\
& \leq \frac{1}{\delta_{\lambda}^{(k), 2}} \sum_{j \neq i}\left\|\bar{v}_{j}^{(k)}\right\|_{0}^{2}\left|\left[v-\mathcal{P}_{k} v, \frac{\bar{v}_{j}^{(k)}}{\left\|\bar{v}_{j}^{(k)}\right\|_{0}}\right]\right|^{2}=\frac{1}{\delta_{\lambda}^{(k), 2}} \sum_{j \neq i} \bar{\mu}_{j}^{(k)}\left|\left[v-\mathcal{P}_{k} v, \frac{\bar{v}_{j}^{(k)}}{\left\|\bar{v}_{j}^{(k)}\right\|_{0}}\right]\right|^{2} \\
& \leq \frac{\bar{\mu}_{1}^{(k)}}{\delta_{\lambda}^{(k), 2}}\left\|v-\mathcal{P}_{k} v\right\|_{0}^{2} .
\end{aligned}
$$

From (3.15), (3.20), and the orthogonal property $\left\langle v-\mathcal{P}_{k} v,\left(I-E_{i, k}\right) \mathcal{P}_{k} v\right\rangle=0$, we have the following error estimates:

$$
\left\|v-E_{i, k} v\right\|^{2}=\left\|v-\mathcal{P}_{k} v\right\|^{2}+\left\|\left(I-E_{i, k}\right) \mathcal{P}_{k} v\right\|^{2}
$$




$$
\leq\left\|\left(I-\mathcal{P}_{k}\right) v\right\|^{2}+\frac{\bar{\mu}_{1}^{(k)}}{\delta_{\lambda}^{(k), 2}}\left\|v-\mathcal{P}_{k} v\right\|_{0}^{2} \leq\left(1+\frac{\bar{\mu}_{1}^{(k)}}{\delta_{\lambda}^{(k), 2}} \eta\left(\mathfrak{V}^{(k)}\right)^{2}\right)\left\|\left(I-\mathcal{P}_{k}\right) v\right\|^{2},
$$

which is the desired result (3.12).

Similarly, combining (3.16), (3.17), (3.18), and (3.19), leads to the following estimates

$$
\begin{aligned}
& \left\|\left(I-E_{i, k}\right) \mathcal{P}_{k} v\right\|_{0}^{2}=\left\|\sum_{j \neq i} \alpha_{j} \bar{v}_{j}^{(k)}\right\|_{0}^{2}=\sum_{j \neq i} \alpha_{j}^{2}\left\|\bar{v}_{j}^{(k)}\right\|_{0}^{2} \\
= & \sum_{j \neq i}\left(\frac{1}{\mu-\bar{\mu}_{j}^{(k)}}\right)^{2}\left|\left[v-\mathcal{P}_{k} v, \bar{v}_{j}^{(k)}\right]\right|^{2}\left\|\bar{v}_{j}^{(k)}\right\|_{0}^{2} \\
\leq & \frac{1}{\delta_{\lambda}^{(k), 2}} \sum_{j \neq i}\left|\left[v-\mathcal{P}_{k} v, \frac{\bar{v}_{j}^{(k)}}{\left\|\bar{v}_{j}^{(k)}\right\|_{0}}\right]\right|^{2}\left\|\bar{v}_{j}^{(k)}\right\|_{0}^{4} \\
= & \frac{1}{\delta_{\lambda}^{(k), 2}} \sum_{j \neq i}\left(\bar{\mu}_{j}^{(k)}\right)^{2}\left|\left[v-\mathcal{P}_{k} v, \frac{\bar{v}_{j}^{(k)}}{\left\|\bar{v}_{j}^{(k)}\right\|_{0}}\right]\right|^{2} \leq\left(\frac{\bar{\mu}_{1}^{(k)}}{\delta_{\lambda}^{(k)}}\right)^{2}\left\|v-\mathcal{P}_{k} v\right\|_{0}^{2} .
\end{aligned}
$$

By (3.15) and (3.21), we have the following inequalities:

$$
\left\|\left(I-E_{i, k}\right) \mathcal{P}_{k} v\right\|_{0} \leq \frac{\bar{\mu}_{1}^{(k)}}{\delta_{\lambda}^{(k)}}\left\|v-\mathcal{P}_{k} v\right\|_{0} \leq \frac{\bar{\mu}_{1}^{(k)}}{\delta_{\lambda}^{(k)}} \eta\left(\mathfrak{V}^{(k)}\right)\left\|\left(I-\mathcal{P}_{k}\right) v\right\| .
$$

From (3.15), (3.22), and the triangle inequality, we conclude that the following error estimate for the eigenvector approximation in $L^{2}$-norm holds:

$$
\begin{aligned}
& \left\|v-E_{i, k} v\right\|_{0} \leq\left\|v-\mathcal{P}_{k} v\right\|_{0}+\left\|\left(I-E_{i, k}\right) \mathcal{P}_{k} v\right\|_{0} \\
\leq & \left\|v-\mathcal{P}_{k} v\right\|_{0}+\frac{\bar{\mu}_{1}^{(k)}}{\delta_{\lambda}^{(k)}} \eta\left(\mathfrak{V}^{(k)}\right)\left\|\left(I-\mathcal{P}_{k}\right) v\right\| \\
\leq & \left(1+\frac{\bar{\mu}_{1}^{(k)}}{\delta_{\lambda}^{(k)}}\right) \eta\left(\mathfrak{V}^{(k)}\right)\left\|\left(I-\mathcal{P}_{k}\right) v\right\| \\
\leq & \left(1+\frac{\bar{\mu}_{1}^{(k)}}{\delta_{\lambda}^{(k)}}\right) \eta\left(\mathfrak{V}^{(k)}\right)\left\|\left(I-E_{i, k}\right) v\right\| .
\end{aligned}
$$

This is the second desired result (3.13) and the proof is complete.

In order to analyze the method which will be given in this section, we state some error estimates in the following lemma.

LEMma 3.5. Under the conditions of Lemma 3.4, the following error estimates hold:

$$
\begin{aligned}
\left\|v-\bar{v}_{i}^{(k)}\right\| & \leq \sqrt{2\left(1+\frac{1}{\lambda_{1} \delta_{\lambda}^{(k), 2}} \eta^{2}\left(\mathfrak{V}^{(k)}\right)\right)}\left\|\left(I-\mathcal{P}_{k}\right) v\right\|, \\
\left\|\lambda v-\bar{\lambda}_{i}^{(k)} \bar{v}_{i}^{(k)}\right\|_{0} & \leq C_{\lambda} \eta\left(\mathfrak{V}^{(k)}\right)\left\|v-\bar{v}_{i}^{(k)}\right\|, \\
\left\|v-\bar{v}_{i}^{(k)}\right\| & \leq \frac{1}{1-D_{\lambda} \eta\left(\mathfrak{V}^{(k)}\right)}\left\|v-\mathcal{P}_{k} v\right\|,
\end{aligned}
$$

Copyright $($ C by SIAM. Unauthorized reproduction of this article is prohibited. 
where

$$
C_{\lambda}=2|\lambda|\left(1+\frac{1}{\lambda_{1} \delta_{\lambda}^{(k)}}\right)+\bar{\lambda}_{i}^{(k)} \sqrt{1+\frac{1}{\lambda_{1} \delta_{\lambda}^{(k), 2}} \eta^{2}\left(\mathfrak{V}^{(k)}\right)^{2}},
$$

and

$$
D_{\lambda}=\frac{1}{\sqrt{\lambda_{1}}}\left(2|\lambda|\left(1+\frac{1}{\lambda_{1} \delta_{\lambda}^{(k)}}\right)+\bar{\lambda}_{i}^{(k)} \sqrt{1+\frac{1}{\lambda_{1} \delta_{\lambda}^{(k), 2}} \eta^{2}\left(\mathfrak{V}^{(k)}\right)}\right) .
$$

Proof. Let us set $\alpha>0$ such that $E_{i, k} v=\alpha \bar{v}_{i}^{(k)}$. Then it implies that

$$
1=\|v\| \geq\left\|E_{i, k} v\right\|=\alpha\left\|\bar{v}_{i}^{(k)}\right\|=\alpha .
$$

Based on the error estimates in Lemma 3.4, the property $\|v\|=\left\|\bar{v}_{i}^{(k)}\right\|=1$ and (3.29), we have the following estimations

$$
\begin{aligned}
& \left\|v-\bar{v}_{i}^{(k)}\right\|^{2}=\left\|v-E_{i, k} v\right\|^{2}+\left\|\bar{v}_{i}^{(k)}-E_{i, k} v\right\|^{2} \\
= & \left\|v-E_{i, k} v\right\|^{2}+\left\|\bar{v}_{i}^{(k)}\right\|^{2}-2\left\langle\bar{v}_{i}^{(k)}, E_{i, k} v\right\rangle+\left\|E_{i, k} v\right\|^{2} \\
= & \left\|v-E_{i, k} v\right\|^{2}+1-2\left\|\bar{v}_{i}^{(k)}\right\|\left\|E_{i, k} v\right\|+\left\|E_{i, k} v\right\|^{2} \\
= & \left\|v-E_{i, k} v\right\|^{2}+\|v\|^{2}-2\|v\|\left\|E_{i, k} v\right\|+\left\|E_{i, k} v\right\|^{2} \\
\leq & \left\|v-E_{i, k} v\right\|^{2}+\|v\|^{2}-2\left\langle v, E_{i, k} v\right\rangle+\left\|E_{i, k} v\right\|^{2} \leq 2\left\|v-E_{i, k} v\right\|^{2} .
\end{aligned}
$$

Equations (3.12) and (3.30) lead to the desired result (3.24).

With the help of (3.13) and the property (3.29) and $\|v\|_{0}=\frac{1}{\sqrt{\lambda}} \geq\left\|\bar{v}_{i}^{(k)}\right\|_{0}=\frac{1}{\sqrt{\bar{\lambda}_{i}^{(k)}}}$, we have the following estimates for $\left\|v-\bar{v}_{i}^{(k)}\right\|_{0}$ :

$$
\begin{aligned}
& \left\|v-\bar{v}_{i}^{(k)}\right\|_{0} \leq\left\|v-E_{i, k} v\right\|_{0}+\left\|E_{i, k} v-\bar{v}_{i}^{(k)}\right\|_{0} \\
= & \left\|v-E_{i, k} v\right\|_{0}+\left\|\bar{v}_{i}^{(k)}\right\|_{0}-\left\|E_{i, k} v\right\|_{0}=\left\|v-E_{i, k} v\right\|_{0}+\frac{1}{\sqrt{\bar{\lambda}_{i}^{(k)}}}-\left\|E_{i, k} v\right\|_{0} \\
\leq & \left\|v-E_{i, k} v\right\|_{0}+\frac{1}{\sqrt{\lambda}}-\left\|E_{i, k} v\right\|_{0}=\left\|v-E_{i, k} v\right\|_{0}+\|v\|_{0}-\left\|E_{i, k} v\right\|_{0} \\
\leq & \left\|v-E_{i, k} v\right\|_{0}+\left\|v-E_{i, k} v\right\|_{0} \leq 2\left\|v-E_{i, k} v\right\|_{0} \\
(3.31) \leq & 2\left(1+\frac{1}{\bar{\lambda}_{1}^{(k)} \delta_{\lambda}^{(k)}}\right) \eta\left(\mathfrak{V}^{(k)}\right)\left\|v-E_{i, k} v\right\| \leq 2\left(1+\frac{1}{\lambda_{1} \delta_{\lambda}^{(k)}}\right) \eta\left(\mathfrak{V}^{(k)}\right)\left\|v-\bar{v}_{i}^{(k)}\right\| .
\end{aligned}
$$

From the expansion (3.10), the definition (3.5), error estimate (3.12), and the property $\left\|\bar{v}_{i}^{(k)}-E \bar{v}_{i}^{(k)}\right\|=\left\|v-E_{i, k} v\right\| \leq\left\|v-\bar{v}_{i}^{(k)}\right\|$, the following error estimates hold:

$$
\begin{aligned}
& \left|\lambda-\bar{\lambda}_{i}^{(k)}\right| \leq \frac{\left\|\bar{v}_{i}^{(k)}-E \bar{v}_{i}^{(k)}\right\|^{2}}{\left\|\bar{v}_{i}^{(k)}\right\|_{0}^{2}}=\frac{\left\|v-E_{i, k} v\right\|^{2}}{\left\|\bar{v}_{i}^{(k)}\right\|_{0}^{2}} \\
\leq & \bar{\lambda}_{i}^{(k)} \sqrt{1+\frac{1}{\lambda_{1} \delta_{\lambda}^{(k), 2}} \eta^{2}\left(\mathfrak{V}^{(k)}\right)\left\|\left(I-\mathcal{P}_{k}\right) v\right\|\left\|v-\bar{v}_{i}^{(k)}\right\|} \\
\leq & \lambda \bar{\lambda}_{i}^{(k)} \sqrt{1+\frac{1}{\lambda_{1} \delta_{\lambda}^{(k), 2}} \eta^{2}\left(\mathfrak{V}^{(k)}\right)}\left\|\left(I-\mathcal{P}_{k}\right) \mathcal{L}^{-1} v\right\|\left\|v-\bar{v}_{i}^{(k)}\right\|
\end{aligned}
$$

Copyright $@$ by SIAM. Unauthorized reproduction of this article is prohibited. 


$$
\begin{aligned}
& \leq \lambda \bar{\lambda}_{i}^{(k)} \sqrt{1+\frac{1}{\lambda_{1} \delta_{\lambda}^{(k), 2}} \eta^{2}\left(\mathfrak{V}^{(k)}\right)} \eta\left(\mathfrak{V}^{(k)}\right)\|v\|_{0}\left\|v-\bar{v}_{i}^{(k)}\right\| \\
& \leq \sqrt{\lambda} \bar{\lambda}_{i}^{(k)} \sqrt{1+\frac{1}{\lambda_{1} \delta_{\lambda}^{(k), 2}} \eta^{2}\left(\mathfrak{V}^{(k)}\right)} \eta\left(\mathfrak{V}^{(k)}\right)\left\|v-\bar{v}_{i}^{(k)}\right\| .
\end{aligned}
$$

Then the combination of (3.31), (3.32), and the property $\left\|\bar{v}_{i}^{(k)}\right\|_{0}=1 / \sqrt{\bar{\lambda}_{i}^{(k)}} \leq$ $1 / \sqrt{\lambda}$ leads to the following estimate:

$$
\begin{aligned}
& \left\|\lambda v-\bar{\lambda}_{i}^{(k)} \bar{v}_{i}^{(k)}\right\|_{0} \leq|\lambda|\left\|v-\bar{v}_{i}^{(k)}\right\|_{0}+\left\|\bar{v}_{i}^{(k)}\right\|_{0} \mid \lambda-\bar{\lambda}_{i}^{(k)} \|_{0} \\
\leq & \left(2|\lambda|\left(1+\frac{1}{\lambda_{1} \delta_{\lambda}^{(k)}}\right)+\left\|\bar{v}_{i}^{(k)}\right\|_{0} \sqrt{\lambda} \bar{\lambda}_{i}^{(k)} \sqrt{1+\frac{1}{\lambda_{1} \delta_{\lambda}^{(k), 2}} \eta\left(\mathfrak{V}^{(k)}\right)^{2}}\right) \eta\left(\mathfrak{V}^{(k)}\right)\left\|v-\bar{v}_{i}^{(k)}\right\| \\
\leq & \left(2|\lambda|\left(1+\frac{1}{\lambda_{1} \delta_{\lambda}^{(k)}}\right)+\bar{\lambda}_{i}^{(k)} \sqrt{1+\frac{1}{\lambda_{1} \delta_{\lambda}^{(k), 2}} \eta^{2}\left(\mathfrak{V}^{(k)}\right)^{2}}\right) \eta\left(\mathfrak{V}^{(k)}\right)\left\|v-\bar{v}_{i}^{(k)}\right\|,
\end{aligned}
$$

which is the desired result (3.25).

We now investigate the distance of $\mathcal{P}_{k} v$ from $\bar{v}_{i}^{(k)}$. First, the following estimate holds:

$$
\begin{aligned}
& \left\|\mathcal{P}_{k} v-\bar{v}_{i}^{(k)}\right\|^{2}=\left\langle\mathcal{P}_{k} v-\bar{v}_{i}^{(k)}, \mathcal{P}_{k} v-\bar{v}_{i}^{(k)}\right\rangle=\left\langle v-\bar{v}_{i}^{(k)}, \mathcal{P}_{k} v-\bar{v}_{i}^{(k)}\right\rangle \\
& =\left[\lambda v-\bar{\lambda}_{i}^{(k)} \bar{v}_{i}^{(k)}, \mathcal{P}_{k} v-\bar{v}_{i}^{(k)}\right] \leq\left\|\lambda v-\bar{\lambda}_{i}^{(k)} \bar{v}_{i}^{(k)}\right\|_{0}\left\|\mathcal{P}_{k} v-\bar{v}_{i}^{(k)}\right\|_{0} \\
& \leq \frac{1}{\sqrt{\lambda_{1}}}\left\|\lambda v-\bar{\lambda}_{i}^{(k)} \bar{v}_{i}^{(k)}\right\|_{0}\left\|\mathcal{P}_{k} v-\bar{v}_{i}^{(k)}\right\| .
\end{aligned}
$$

From (3.33) and (3.34), we have the following estimate:

$$
\begin{aligned}
& \left\|\mathcal{P}_{k} v-\bar{v}_{i}^{(k)}\right\| \leq \frac{1}{\sqrt{\lambda_{1}}}\left\|\lambda v-\bar{\lambda}_{i}^{(k)} \bar{v}_{i}^{(k)}\right\|_{0} \\
& 3.35) \leq \frac{1}{\sqrt{\lambda_{1}}}\left(2|\lambda|\left(1+\frac{1}{\lambda_{1} \delta_{\lambda}^{(k)}}\right)+\bar{\lambda}_{i}^{(k)} \sqrt{1+\frac{\bar{\mu}_{1}^{(k)}}{\delta_{\lambda}^{(k), 2}} \eta\left(\mathfrak{V}^{(k)}\right)^{2}}\right) \eta\left(\mathfrak{V}^{(k)}\right)\left\|v-\bar{v}_{i}^{(k)}\right\| .
\end{aligned}
$$

Equation (3.35) and the triangle inequality lead to the following inequality:

$$
\begin{aligned}
& \left\|v-\bar{v}_{i}^{(k)}\right\| \leq\left\|v-\mathcal{P}_{k} v\right\|+\left\|\mathcal{P}_{k} v-\bar{v}_{i}^{(k)}\right\| \\
& \leq\left\|v-\mathcal{P}_{k} v\right\| \\
& +\frac{1}{\sqrt{\lambda_{1}}}\left(2|\lambda|\left(1+\frac{1}{\lambda_{1} \delta_{\lambda}^{(k)}}\right)+\bar{\lambda}_{i}^{(k)} \sqrt{1+\frac{\bar{\mu}_{1}^{(k)}}{\delta_{\lambda}^{(k), 2}} \eta^{2}\left(\mathfrak{V}^{(k)}\right)}\right) \eta\left(\mathfrak{V}^{(k)}\right)\left\|v-\bar{v}_{i}^{(k)}\right\|,
\end{aligned}
$$

which in turn implies that

$$
\begin{aligned}
& \left\|v-\bar{v}_{i}^{(k)}\right\| \\
& \leq \frac{1}{1-\frac{1}{\sqrt{\lambda_{1}}}\left(2|\lambda|\left(1+\frac{1}{\lambda_{1} \delta_{\lambda}^{(k)}}\right)+\bar{\lambda}_{i}^{(k)} \sqrt{1+\frac{1}{\lambda_{1} \delta_{\lambda}^{(k), 2}} \eta^{2}\left(\mathfrak{V}^{(k)}\right)}\right) \eta\left(\mathfrak{V}^{(k)}\right)}\left\|v-\mathcal{P}_{k} v\right\|
\end{aligned}
$$

Copyright $@$ by SIAM. Unauthorized reproduction of this article is prohibited. 


$$
\leq \frac{1}{1-D_{\lambda} \eta\left(\mathfrak{V}^{(k)}\right)}\left\|v-\mathcal{P}_{k} v\right\|
$$

This completes the proof of the desired result (3.26).

3.1. One correction step. To describe the multilevel correction method we first present the "one correction step." Given an eigenpair approximation $\left(\lambda^{(k, \ell)}, v^{(k, \ell)}\right)$ $\in \mathbb{R} \times \mathfrak{V}^{(k)}$, Algorithm 3.1 produces an improved eigenpair approximation $\left(\lambda^{(k, \ell+1)}\right.$, $\left.v^{(k, \ell+1)}\right) \in \mathbb{R} \times \mathfrak{V}^{(k)}$. In this algorithm, the superscript $(k, \ell)$ denotes the $\ell$ th correction step in the $k$ th level gamblet space.

Algorithm 3.1 One correction step.

1. Let $\widetilde{v}^{(k, \ell+1)} \in \mathfrak{V}^{(k)}$ be the solution of the linear system

$$
\left\langle\widetilde{v}^{(k, \ell+1)}, w\right\rangle=\lambda^{(k, \ell)}\left[v^{(k, \ell)}, w\right] \quad \forall w \in \mathfrak{V}^{(k)} .
$$

Approximate $\widetilde{v}^{(k, \ell+1)}$ by $\widehat{v}^{(k, \ell+1)}=\operatorname{MG}\left(k, v^{(k, \ell)}, \lambda^{(k, \ell)} v^{(k, \ell)}\right)$ using Algorithm 2.2 .

2. Let $\mathfrak{V}^{(1)}$ be the coarsest gamblet space, define

$$
\mathfrak{V}^{(1, k)}=\mathfrak{V}^{(1)}+\operatorname{span}\left\{\widehat{v}^{(k, \ell+1)}\right\},
$$

and solve the subspace eigenvalue problem: Find $\left(\lambda^{(k, \ell+1)}, v^{(k, \ell+1)}\right) \in \mathbb{R} \times$ $\mathfrak{V}^{(1, k)}$ such that $\left\langle v^{(k, \ell+1)}, v^{(k, \ell+1)}\right\rangle=1$ and

$$
\left\langle v^{(k, \ell+1)}, w\right\rangle=\lambda^{(k, \ell+1)}\left[v^{(k, \ell+1)}, w\right] \quad \forall w \in \mathfrak{V}^{(1, k)} .
$$

Let EigenMG be the function summarizing the action of the steps described above, i.e.,

$$
\left(\lambda^{(k, \ell+1)}, v^{(k, \ell+1)}\right)=\operatorname{EigenMG}\left(\mathfrak{V}^{(1)}, \lambda^{(k, \ell)}, v^{(k, \ell)}, \mathfrak{V}^{(k)}\right) .
$$

Remark 3.6. Notice that in (3.37), the orthogonalization is only performed in the coarse space $\mathfrak{V}^{(1, k)}$ with dimension $1+\operatorname{dim} \mathfrak{V}^{(1)}$.

For simplicity of notation, we assume that the eigenvalue gap $\delta_{\lambda}^{(k)}$ has a uniform lower bound which is denoted by $\delta_{\lambda}$ (which can be seen as the "true" separation of the eigenvalues) in the following parts of this paper. This assumption is reasonable when the mesh size $H$ is small enough. We refer to [47, Theorem 4.6] for details on the dependence of error estimates on the eigenvalue gap. Furthermore, we also assume the concerned eigenpair approximation $\left(\lambda^{(k, \ell)}, v^{(k, \ell)}\right)$ is closet to the exact eigenpair $\left(\bar{\lambda}^{(k)}, \bar{v}^{(k)}\right)$ of $(3.3)$ and $(\lambda, v)$ of $(3.1)$.

TheOREm 3.7. Assume there exists exact eigenpair $\left(\bar{\lambda}^{(k)}, \bar{v}^{(k)}\right)$ such that the eigenpair approximation $\left(\lambda^{(k, \ell)}, v^{(k, \ell)}\right)$ satisfies $\left\|v^{(k, \ell)}\right\|=1$ and

$$
\left\|\bar{\lambda}^{(k)} \bar{v}^{(k)}-\lambda^{(k, \ell)} v^{(k, \ell)}\right\|_{0} \leq C_{1} \eta\left(\mathfrak{V}^{(1)}\right)\left\|\bar{v}^{(k)}-v^{(k, \ell)}\right\|
$$

for some constant $C_{1}$. The multigrid iteration for the linear equation (3.36) has the following uniform contraction rate:

$$
\left\|\widehat{v}^{(k, \ell+1)}-\widetilde{v}^{(k, \ell+1)}\right\| \leq \theta\left\|v^{(k, \ell)}-\widetilde{v}^{(k, \ell+1)}\right\|
$$

Copyright $@$ by SIAM. Unauthorized reproduction of this article is prohibited. 
with $\theta<1$ independent from $k$ and $\ell$.

Then the eigenpair approximation $\left(\lambda^{(k, \ell+1)}, v^{(k, \ell+1)}\right) \in \mathbb{R} \times \mathfrak{V}^{(k)}$ produced by Algorithm 3.1 satisfies

$$
\begin{aligned}
\left\|\bar{v}^{(k)}-v^{(k, \ell+1)}\right\| & \leq \gamma\left\|\bar{v}^{(k)}-v^{(k, \ell)}\right\|, \\
\left\|\bar{\lambda}^{(k)} \bar{v}^{(k)}-\lambda^{(k, \ell+1)} v^{(k, \ell+1)}\right\|_{0} & \leq \bar{C}_{\lambda} \eta\left(\mathfrak{V}^{(1)}\right)\left\|\bar{v}^{(k)}-v^{(k, \ell+1)}\right\|,
\end{aligned}
$$

where the constants $\gamma, \bar{C}_{\lambda}$, and $D_{\lambda}$ are defined as follows:

$$
\begin{gathered}
\gamma=\frac{1}{1-\bar{D}_{\lambda} \eta\left(\mathfrak{V}^{(1)}\right)}\left(\theta+(1+\theta) \frac{C_{1}}{\sqrt{\lambda_{1}}} \eta\left(\mathfrak{V}^{(1)}\right)\right), \\
\bar{C}_{\lambda}=2|\lambda|\left(1+\frac{1}{\lambda_{1} \delta_{\lambda}}\right)+\bar{\lambda}_{i}^{(1)} \sqrt{1+\frac{1}{\lambda_{1} \delta_{\lambda}^{2}} \eta^{2}\left(\mathfrak{V}^{(1)}\right)^{2}}, \\
\bar{D}_{\lambda}=\frac{1}{\sqrt{\lambda_{1}}}\left(2|\lambda|\left(1+\frac{1}{\lambda_{1} \delta_{\lambda}}\right)+\bar{\lambda}_{i}^{(1)} \sqrt{1+\frac{1}{\lambda_{1} \delta_{\lambda}^{2}} \eta^{2}\left(\mathfrak{V}^{(1)}\right)}\right) .
\end{gathered}
$$

Proof. From (3.2), (3.3), and (3.36), we have for $w \in \mathfrak{V}^{(k)}$,

$$
\begin{aligned}
& \left\langle\bar{v}^{(k)}-\widetilde{v}^{(k, \ell+1)}, w\right\rangle=\left[\left(\bar{\lambda}^{(k)} \bar{v}^{(k)}-\lambda^{(k, \ell)} v^{(k, \ell)}\right), w\right] \\
\leq & \left\|\bar{\lambda}^{(k)} \bar{v}^{(k)}-\lambda^{(k, \ell)} v^{(k, \ell)}\right\|_{0}\|w\|_{0} \leq C_{1} \eta\left(\mathfrak{V}^{(k)}\right)\left\|\bar{v}^{(k)}-v^{(k, \ell)}\right\|\|w\|_{0} \\
\leq & \frac{1}{\sqrt{\lambda_{1}}} C_{1} \eta\left(\mathfrak{V}^{(k)}\right)\left\|\bar{v}^{(k)}-v^{(k, \ell)}\right\|\|w\| .
\end{aligned}
$$

Taking $w=\bar{v}^{(k)}-\widetilde{v}^{(k, \ell+1)}$ we deduce from (3.38) that

$$
\left\|\bar{v}^{(k)}-\widetilde{v}^{(k, \ell+1)}\right\| \leq \frac{C_{1}}{\sqrt{\lambda_{1}}} \eta\left(\mathfrak{V}^{(1)}\right)\left\|\bar{v}^{(k)}-v^{(k, \ell)}\right\| .
$$

Using (3.39) and (3.45) we deduce that

$$
\begin{aligned}
\left\|\bar{v}^{(k)}-\widehat{v}^{(k, \ell+1)}\right\| & \leq\left\|\bar{v}^{(k)}-\widetilde{v}^{(k, \ell+1)}\right\|+\left\|\widetilde{v}^{(k, \ell+1)}-\widehat{v}^{(k, \ell+1)}\right\| \\
& \leq\left\|\bar{v}^{(k)}-\widetilde{v}^{(k, \ell+1)}\right\|+\theta\left\|\widetilde{v}^{(k, \ell+1)}-v^{(k, \ell)}\right\| \\
& \leq\left\|\bar{v}^{(k)}-\widetilde{v}^{(k, \ell+1)}\right\|+\theta\left\|\widetilde{v}^{(k, \ell+1)}-\bar{v}^{(k)}\right\|+\theta\left\|\bar{v}^{(k)}-v^{(k, \ell)}\right\| \\
& \leq(1+\theta)\left\|\bar{v}^{(k)}-\widetilde{v}^{(k, \ell+1)}\right\|+\theta\left\|\bar{v}^{(k)}-v^{(k, \ell)}\right\| \\
& \leq\left(\theta+(1+\theta) \frac{C_{1}}{\sqrt{\lambda_{1}}} \eta\left(\mathfrak{V}^{(1)}\right)\right)\left\|\bar{v}^{(k)}-v^{(k, \ell)}\right\| .
\end{aligned}
$$

The eigenvalue problem (3.37) can be seen as a low-dimensional subspace approximation of the eigenvalue problem (3.3). Using (3.26), Lemmas 3.4, 3.5, and their proof, we obtain that

$$
\begin{aligned}
\left\|\bar{v}^{(k)}-v^{(k, \ell+1)}\right\| & \leq \frac{1}{1-\bar{D}_{\lambda} \eta\left(\mathfrak{V}^{(1, k)}\right)} \inf _{w^{(1, k)} \in \mathfrak{V}^{(1, k)}}\left\|\bar{v}^{(k)}-w^{(1, k)}\right\| \\
& \leq \frac{1}{1-\bar{D}_{\lambda} \eta\left(\mathfrak{V}^{(1)}\right)}\left\|\bar{v}^{(k)}-\widehat{v}^{(k, \ell+1)}\right\| \\
& \leq \gamma\left\|\bar{v}^{(k)}-v^{(k, \ell)}\right\|
\end{aligned}
$$

Copyright $@$ by SIAM. Unauthorized reproduction of this article is prohibited. 
and

$$
\begin{aligned}
\left\|\bar{\lambda}^{(k)} \bar{v}^{(k)}-\lambda^{(k, \ell+1)} v^{(k, \ell+1)}\right\|_{0} & \leq \bar{C}_{\lambda} \eta\left(\mathfrak{V}^{(1, k)}\right)\left\|\bar{v}^{(k)}-v^{(k, \ell+1)}\right\| \\
& \leq \bar{C}_{\lambda} \eta\left(\mathfrak{V}^{(1)}\right)\left\|\bar{v}^{(k)}-v^{(k, \ell+1)}\right\| .
\end{aligned}
$$

Then we have the desired results (3.40) and (3.41) and conclude the proof.

Remark 3.8. Definition (3.42), Theorem 2.10, and Lemmas 3.4 and 3.5 imply that $\gamma$ is less than 1 when $\eta\left(\mathfrak{V}^{(1)}\right)$ is small enough. If $\lambda$ is large or the spectral gap $\delta_{\lambda}$ is small, then we need to use a smaller $\eta\left(\mathfrak{V}^{(1)}\right)$ or $H$. Furthermore, we can increase the multigrid smoothing steps $m_{1}$ and $m_{2}$ to reduce $\theta$ and then $\gamma$. These theoretical restrictions do not limit practical applications where (in numerical implementations), $H$ is simply chosen (just) small enough so that the number of elements of corresponding coarsest space (just) exceeds the required number of eigenpairs ( $H$ and the coarsest space are adapted to the number of eigenpairs to be computed).

3.2. Multilevel method for eigenvalue problem. In this subsection, we introduce the multilevel method based on the subspace correction method defined in Algorithm 3.1 and the properties of gamblet spaces. This multilevel method can achieve the same order of accuracy as the direct solve of the eigenvalue problem on the finest (gamblet) space. The multilevel method is presented in Algorithm 3.2.

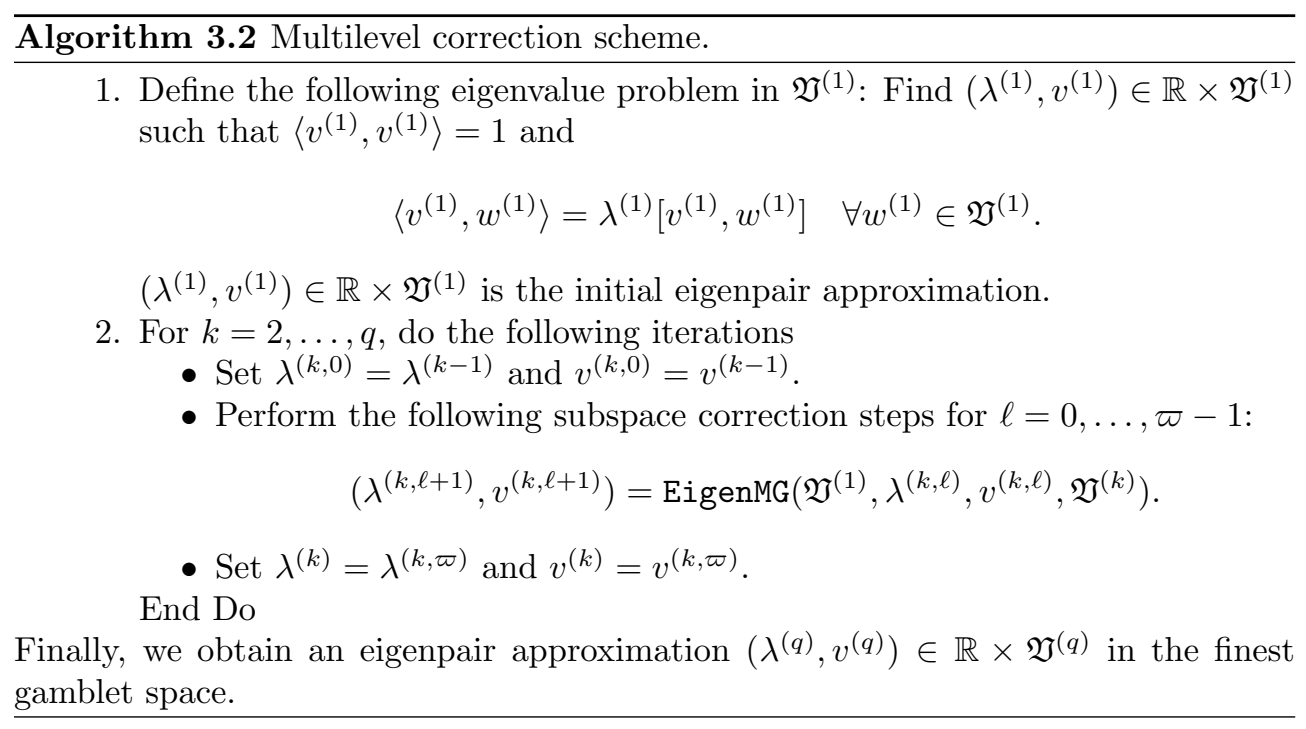

TheOREm 3.9. After implementing Algorithm 3.2, the resulting eigenpair approximation $\left(\lambda^{(q)}, v^{(q)}\right)$ has the following error estimates:

$$
\begin{aligned}
\left\|\bar{v}^{(q)}-v^{(q)}\right\| & \leq 2 \sum_{k=1}^{q-1} \gamma^{(q-k) \varpi} \delta_{k}(\lambda), \\
\left\|\bar{v}^{(q)}-v^{(q)}\right\|_{0} & \leq 2\left(1+\frac{1}{\lambda_{1} \delta_{\lambda}}\right) \eta\left(\mathfrak{V}^{(1)}\right)\left\|\bar{v}^{(q)}-v^{(q)}\right\|, \\
\left|\bar{\lambda}^{(q)}-\lambda^{(q)}\right| & \leq \lambda^{(q)}\left\|v^{(q)}-\bar{v}^{(q)}\right\|^{2},
\end{aligned}
$$

where $\varpi$ is the number of subspace correction steps in Algorithm 3.2 .

Copyright (c) by SIAM. Unauthorized reproduction of this article is prohibited. 
Proof. Define $e_{k}:=\bar{v}^{(k)}-v^{(k)}$. From step 1 in Algorithm 3.2, it is obvious $e_{1}=0$. Then the assumption (3.38) in Theorem 3.7 is satisfied for $k=1$. From the definitions of Algorithms 3.1 and 3.2, Theorem 3.7, and the recursive argument, the assumption (3.38) holds for each level of space $\mathfrak{V}^{(k)}(k=1, \ldots, q)$ with $C_{1}=\bar{C}_{\lambda}$ in (3.43). Then the convergence rate (3.40) is valid for all $k=1, \ldots, q$ and $\ell=0, \ldots, \varpi-1$.

For $k=2, \ldots, q$, by Theorem 3.7 and the recursive argument, we have

$$
\begin{aligned}
\left\|e_{k}\right\| & \leq \gamma^{\varpi}\left\|\bar{v}^{(k)}-v^{(k-1)}\right\| \\
& \leq \gamma^{\varpi}\left(\left\|\bar{v}^{(k)}-\bar{v}^{(k-1)}\right\|+\left\|\bar{v}^{(k-1)}-v^{(k-1)}\right\|\right) \\
& \leq \gamma^{\varpi}\left(\left\|\bar{v}^{(k)}-v\right\|+\left\|v-\bar{v}^{(k-1)}\right\|+\left\|\bar{v}^{(k-1)}-v^{(k-1)}\right\|\right) \\
& =\gamma^{\varpi}\left(\delta_{k}(\lambda)+\delta_{k-1}(\lambda)+\left\|e_{k-1}\right\|\right) \\
& \leq \gamma^{\varpi}\left(2 \delta_{k-1}(\lambda)+\left\|e_{k-1}\right\|\right) .
\end{aligned}
$$

By iterating inequality (3.52), the following inequalities hold:

$$
\left\|e_{q}\right\| \leq 2\left(\gamma^{\varpi} \delta_{q-1}(\lambda)+\cdots+\gamma^{(q-1) \varpi} \delta_{1}(\lambda)\right) \leq 2 \sum_{k=1}^{q-1} \gamma^{(q-k) \varpi} \delta_{k}(\lambda),
$$

which leads to the desired result (3.49).

From (3.10), (3.31), (3.32), and (3.49), we have the following error estimates:

$$
\begin{aligned}
\left\|\bar{v}^{(q)}-v^{(q)}\right\|_{0} & \leq 2\left(1+\frac{1}{\lambda_{1} \delta_{\lambda}}\right) \eta\left(\mathfrak{V}^{(1)}\right)\left\|\bar{v}^{(q)}-v^{(q)}\right\|, \\
\left|\bar{\lambda}^{(q)}-\lambda^{(q)}\right| & \leq \frac{\left\|v^{(q)}-\bar{v}^{(q)}\right\|^{2}}{\left\|v^{(q)}\right\|_{0}^{2}} \leq \lambda^{(q)}\left\|v^{(q)}-\bar{v}^{(q)}\right\|^{2},
\end{aligned}
$$

which are the desired results (3.50) and (3.51).

Remark 3.10. The proof of Theorem 3.9 implies that the assumption (3.38) in Theorem 3.7 holds for $C_{1}=\bar{C}_{\lambda}$ in each level of space $\mathfrak{V}^{(k)}(k=1, \ldots, q)$. The structure of Algorithm 3.2 implies that $\bar{C}_{\lambda}$ does not change as the algorithm progresses from the initial space $\mathfrak{V}^{(1)}$ to the finest one $\mathfrak{V}^{(q)}$.

COROLlary 3.11. Let $\gamma$ be the constant in (3.42). Given the uniform contraction rate $0<\theta<1$ (obtained from Theorem 2.10) and given the bound $\eta\left(\mathfrak{V}^{(1)}\right) \leq C H$ (obtained from Property 2.3, which is implied by Theorem 2.5), select $0<\bar{H}<1$ small enough so that $0<\gamma<1$ and then choose the integer $\varpi>1$ to satisfy

$$
\frac{\gamma^{\varpi}}{H}<1
$$

Then the resulting eigenpair approximation $\left(\lambda^{(q)}, v^{(q)}\right)$ obtained by Algorithm 3.2 has the following error estimates:

$$
\begin{aligned}
\left\|v-v^{(q)}\right\| & \leq C C_{\lambda}^{\prime} \sqrt{\lambda} H^{q}, \\
\left\|v-v^{(q)}\right\|_{0} & \leq 2 C^{2}\left(\left(1+\frac{1}{\lambda_{1} \delta_{\lambda}}\right)\left(1+H^{q-1}\right)\right) C_{\lambda}^{\prime} H^{q}, \\
\left|\lambda-\lambda^{(q)}\right| & \leq \lambda \lambda^{(q)}\left(C C_{\lambda}^{\prime}\right)^{2} H^{2 q},
\end{aligned}
$$

Copyright $@$ by SIAM. Unauthorized reproduction of this article is prohibited. 
where the constant $C$ comes from Property 2.3 or Proposition 3.1 and $C_{\lambda}^{\prime}$ is defined as follows:

$$
C_{\lambda}^{\prime}=\left(\sqrt{2 \lambda\left(1+\frac{1}{\lambda_{1} \delta_{\lambda}^{2}} \eta^{2}\left(\mathfrak{V}^{(q)}\right)\right)}+2 \frac{1-\left(\frac{\gamma^{\varpi}}{H}\right)^{q}}{1-\frac{\gamma^{\varpi}}{H}}\right) .
$$

Proof. From Lemma 3.5, Theorem 3.9, (3.8), (3.24), and (3.54), we have the following estimates

$$
\begin{aligned}
& \left\|v-v^{(q)}\right\| \leq\left\|v-\bar{v}^{(q)}\right\|+\left\|\bar{v}^{(q)}-v^{(q)}\right\| \\
\leq & \sqrt{2\left(1+\frac{1}{\lambda_{1} \delta_{\lambda}^{2}} \eta^{2}\left(\mathfrak{V}^{(q)}\right)\right)} \delta_{q}(\lambda)+2 \sum_{k=1}^{q-1} \gamma^{(q-k) \varpi} \delta_{k}(\lambda) \\
\leq & C \sqrt{2\left(1+\frac{1}{\lambda_{1} \delta_{\lambda}^{2}} \eta^{2}\left(\mathfrak{V}^{(q)}\right)\right)} \sqrt{\lambda} H^{q}+2 C \sum_{k=1}^{q-1} \gamma^{(q-k) \varpi \sqrt{\lambda}} H^{k} \\
\leq & C \sqrt{2 \lambda\left(1+\frac{1}{\lambda_{1} \delta_{\lambda}^{2}} \eta^{2}\left(\mathfrak{V}^{(q)}\right)\right)} \sqrt{\lambda} H^{q}+2 C \sqrt{\lambda} H^{q} \sum_{k=0}^{q-1}\left(\frac{\gamma^{\varpi}}{H}\right)^{k} \\
\leq & C\left(\sqrt{2 \lambda\left(1+\frac{1}{\lambda_{1} \delta_{\lambda}^{2}} \eta^{2}\left(\mathfrak{V}^{(q)}\right)\right)}+2 \frac{1-\left(\frac{\gamma^{\varpi}}{H}\right)^{q}}{1-\frac{\gamma^{\varpi}}{H}}\right) \sqrt{\lambda} H^{q} .
\end{aligned}
$$

This is the desired result (3.55).

From (3.8), (3.31), (3.49), (3.50), and (3.58), $\left\|v-v^{(q)}\right\|_{0}$ has the following estimates:

$$
\begin{aligned}
& \left\|v-v^{(q)}\right\|_{0} \leq\left\|v-\bar{v}^{(q)}\right\|_{0}+\left\|\bar{v}^{(q)}-v^{(q)}\right\|_{0} \\
& \leq 2\left(1+\frac{1}{\lambda_{1} \delta_{\lambda}}\right) \eta\left(\mathfrak{V}^{(q)}\right)\left\|v-\bar{v}^{(q)}\right\|+2\left(1+\frac{1}{\lambda_{1} \delta_{\lambda}}\right) \eta\left(\mathfrak{V}^{(1)}\right)\left\|\bar{v}^{(q)}-v^{(q)}\right\| \\
& \leq 2 C\left(1+\frac{1}{\lambda_{1} \delta_{\lambda}}\right) \eta\left(\mathfrak{V}^{(q)}\right) \sqrt{2\left(1+\frac{1}{\lambda_{1} \delta_{\lambda}^{2}} \eta^{2}\left(\mathfrak{V}^{(q)}\right)\right)} H^{q} \\
& +4 C\left(1+\frac{1}{\lambda_{1} \delta_{\lambda}}\right) \eta\left(\mathfrak{V}^{(1)}\right) \frac{1-\left(\frac{\gamma^{\varpi}}{H}\right)^{q}}{1-\frac{\gamma^{\varpi}}{H}} H^{q} \\
& \leq 2 C\left(\left(1+\frac{1}{\lambda_{1} \delta_{\lambda}}\right)\left(1+H^{q-1}\right)\right) C_{\lambda}^{\prime} H^{q} .
\end{aligned}
$$

From (3.10) and (3.55), the error estimate for $\left|\lambda-\lambda^{(q)}\right|$ can be deduced as follows:

$$
\left|\lambda-\lambda^{(q)}\right| \leq \frac{\left\|v^{(q)}-v\right\|^{2}}{\left\|v^{(q)}\right\|_{0}^{2}} \leq \lambda^{(q)}\left\|v^{(q)}-v\right\|^{2} \leq \lambda \lambda^{(q)} C^{2} C_{\lambda}^{\prime 2} H^{2 q} .
$$

Then the desired results (3.56) and (3.57) are obtained and the proof is complete.

Remark 3.12. The main computational work of Algorithm 3.1 is to solve the linear equation (3.36) by the multigrid method defined in Algorithm 2.2. Therefore, Remark 2.9 implies the bound $\mathcal{O}\left(N\left(\log \left(\frac{N}{\varepsilon}\right)\right)^{2 d+1} \log (\varepsilon) / \log (\gamma)\right)$ on the number of operations required to achieve accuracy $\varepsilon$ (see $[41,42,44,48,43])$.

Copyright (c) by SIAM. Unauthorized reproduction of this article is prohibited. 
4. Numerical results. In this section, numerical examples are presented to illustrate the efficiency of the gamblet based multilevel correction method for benchmark multiscale eigenvalue problems. Furthermore, we will show that the gamblets can also be used as efficient preconditioner for state-of-the-art eigensolvers such as LOBPCG method.

4.1. SPE10. In the first example, we solve the eigenvalue problem (2.8) on $\Omega=$ $[-1,1] \times[-1,1]$, and the coefficient matrix $a(x)$ is taken from the data of the SPE10 benchmark (http://www.spe.org/web/csp/). The contrast of $a(x)$ is $\lambda_{\max }(a) / \lambda_{\min }(a)$ $\simeq 1 \cdot 10^{6}$.

The fine mesh $\mathcal{T}_{h}$ is a regular square mesh with mesh size $h=2\left(1+2^{q}\right)^{-1}$ and $128 \times 128$ interior nodes. At the finest level, we use continuous bilinear nodal basis elements $\varphi_{i}$ spanned by $\left\{1, x_{1}, x_{2}, x_{1} x_{2}\right\}$ in each element of $\mathcal{T}_{h} . a(x)$ is piecewise constant over $\mathcal{T}_{h}$ as illustrated in Figure 2. The measurement function is chosen as in Example 2.4. For the gamblet decomposition, we choose $H=1 / 2, q=7$. The prewavelets $\psi$ and the gamblet decomposition of the solution $u$ for the elliptic equation $-\operatorname{div} a(x) \nabla u=\sin (\pi x) \sin (\pi y)$ are shown in Figures 3 and 4, respectively.
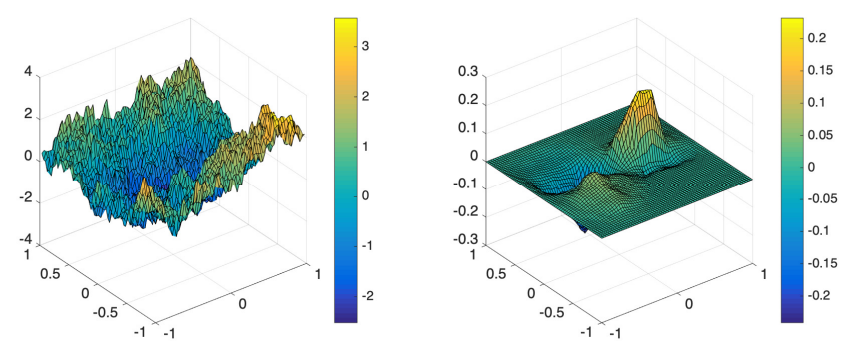

FIG. 2. Left: coefficient a $(x)$ from SPE10 benchmark, in $\log _{10}$ scale. Right: solution $u$ for the elliptic equation $-\operatorname{div} a(x) \nabla u=\sin (\pi x) \sin (\pi y)$.

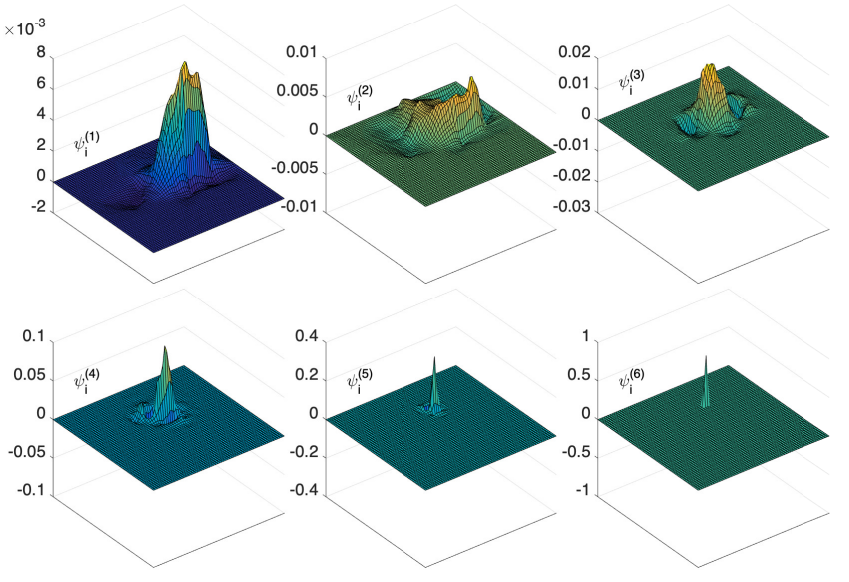

FiG. 3. Prewavelets $\psi$ at different scales. 


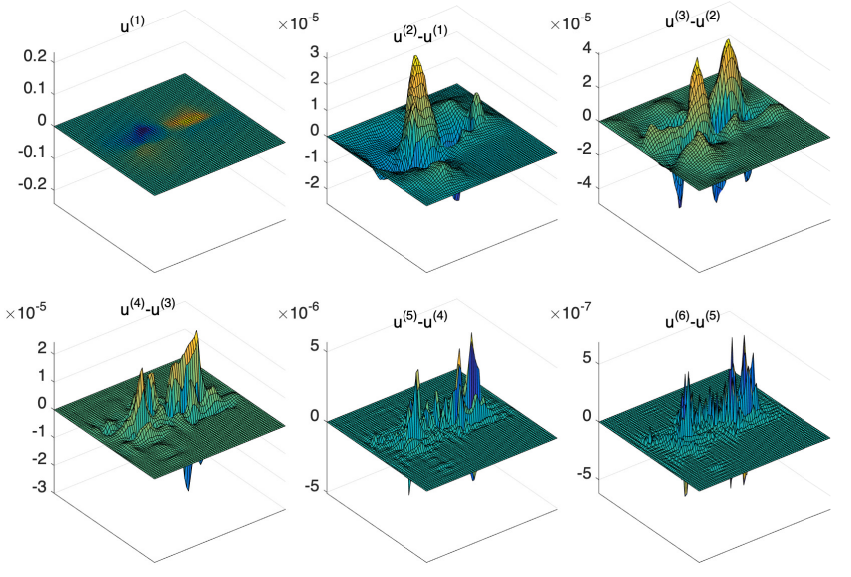

FIG. 4. Solution for the elliptic equation with $f=\sin (\pi x) \sin (\pi y)$.

We calculate the first 12 eigenvalues using the multilevel correction method in Algorithm 3.2; therefore, we actually take $\mathfrak{V}^{(2)}$ as the coarsest subspace and the effective mesh size is $H^{2}=1 / 4$. We choose parameters $m_{1}=m_{2}=2$ and $p=1$ in the multigrid iteration step defined in Algorithm 2.2 to solve the linear equation (3.36), and use Gauss-Seidel as the smoother.

We compare the gamblet based multilevel correction method with geometric multigrid multilevel correction method. In Table 1, we show the numerical results for the first 12 eigenvalues. Here we take the number of subspace correction steps $\varpi=1$ for $k=3, \ldots, q$. For comparison, we also show the corresponding numerical results in Table 2 with the standard geometric multigrid linear solver. We observe much faster convergence for the gamblet based multilevel correction method $\left(10^{6}\right.$ smaller for the first eigenvalue).

TABLE 1

Relative errors $\left|\left(\lambda_{i}^{(k)}-\lambda_{i}\right) / \lambda_{i}\right|$ for the gamblet based multilevel correction method. First, a few iterations on the coarser levels.

\begin{tabular}{|c|c|c|c|c|c|c|}
\hline $\mathrm{i}$ & $\mathrm{k}=2$ & $\mathrm{k}=3$ & $\mathrm{k}=4$ & $\mathrm{k}=5$ & $\mathrm{k}=6$ & $\mathrm{k}=7$ \\
\hline 1 & $6.1568 \mathrm{e}-2$ & $1.3356 \mathrm{e}-2$ & $3.0902 \mathrm{e}-3$ & $1.2586 \mathrm{e}-3$ & $3.8293 \mathrm{e}-4$ & $1.5586 \mathrm{e}-8$ \\
2 & $1.6827 \mathrm{e}-1$ & $3.0270 \mathrm{e}-2$ & $4.6347 \mathrm{e}-3$ & $1.0656 \mathrm{e}-3$ & $2.4616 \mathrm{e}-4$ & $5.3456 \mathrm{e}-8$ \\
3 & $7.9106 \mathrm{e}-1$ & $1.1814 \mathrm{e}-1$ & $2.3155 \mathrm{e}-2$ & $2.8431 \mathrm{e}-3$ & $2.9124 \mathrm{e}-4$ & $4.7883 \mathrm{e}-6$ \\
4 & $5.8274 \mathrm{e}-1$ & $1.9203 \mathrm{e}-1$ & $4.5203 \mathrm{e}-2$ & $7.7621 \mathrm{e}-3$ & $7.7980 \mathrm{e}-4$ & $4.4444 \mathrm{e}-5$ \\
5 & $7.5657 \mathrm{e}-1$ & $1.6533 \mathrm{e}-1$ & $1.6978 \mathrm{e}-2$ & $2.8863 \mathrm{e}-3$ & $3.3941 \mathrm{e}-4$ & $1.0250 \mathrm{e}-5$ \\
6 & $9.4417 \mathrm{e}-1$ & $2.9132 \mathrm{e}-1$ & $5.0443 \mathrm{e}-2$ & $7.0754 \mathrm{e}-3$ & $7.7061 \mathrm{e}-4$ & $4.4771 \mathrm{e}-5$ \\
7 & $1.7033 \mathrm{e} 0$ & $2.8337 \mathrm{e}-1$ & $8.1393 \mathrm{e}-2$ & $2.4187 \mathrm{e}-2$ & $4.6014 \mathrm{e}-3$ & $7.2897 \mathrm{e}-4$ \\
8 & $2.4517 \mathrm{e} 0$ & $5.0598 \mathrm{e}-1$ & $1.3164 \mathrm{e}-1$ & $2.4945 \mathrm{e}-2$ & $4.6447 \mathrm{e}-3$ & $8.7663 \mathrm{e}-4$ \\
9 & $6.4576 \mathrm{e} 0$ & $6.6654 \mathrm{e}-1$ & $2.6205 \mathrm{e}-1$ & $9.9177 \mathrm{e}-2$ & $1.6962 \mathrm{e}-2$ & $3.3086 \mathrm{e}-3$ \\
10 & $6.9955 \mathrm{e} 0$ & $6.8507 \mathrm{e}-1$ & $2.4108 \mathrm{e}-1$ & $4.7575 \mathrm{e}-2$ & $1.9529 \mathrm{e}-2$ & $9.6051 \mathrm{e}-3$ \\
11 & $1.0927 \mathrm{e} 1$ & $8.6987 \mathrm{e}-1$ & $2.6043 \mathrm{e}-1$ & $7.5851 \mathrm{e}-2$ & $1.9996 \mathrm{e}-2$ & $8.3358 \mathrm{e}-3$ \\
12 & $1.3665 \mathrm{e} 1$ & $9.5975 \mathrm{e}-1$ & $3.3355 \mathrm{e}-1$ & $5.9182 \mathrm{e}-2$ & $1.9377 \mathrm{e}-2$ & $7.5015 \mathrm{e}-3$ \\
\hline
\end{tabular}

Remark 4.1. It is shown in [37] that for approximate eigenvalues with respect to the LOD coarse spaces on scale $H$, a postprocessing step can improve the eigenvalue error from $H^{4}$ to $H^{6}$. The postprocessing step is a correction with exact solve on the finest level. Since we are using an approximate solve in the correction step, this corresponds to the multilevel correction scheme with one correction step on each level, which is shown in Table 1. Comparing Table 1 with Table 2 in [37] shows a similar 
improvement of accuracy at the finer levels (although the coefficients $a(x)$ are not the same, we expect a similar behavior for the approximation errors of eigenvalues). However, with geometric multigrid, the error reduction is very slow, which is shown by Table 2 .

TABLE 2

Relative errors $\left|\left(\lambda_{i}^{(k)}-\lambda_{i}\right) / \lambda_{i}\right|$ for multilevel correction with geometric multigrid. First, a few iterations on the coarser levels.

\begin{tabular}{|c|c|c|c|c|c|c|}
\hline $\mathrm{i}$ & $\mathrm{k}=2$ & $\mathrm{k}=3$ & $\mathrm{k}=4$ & $\mathrm{k}=5$ & $\mathrm{k}=6$ & $\mathrm{k}=7$ \\
\hline 1 & $2.6912 \mathrm{e} 0$ & $2.6698 \mathrm{e} 0$ & $2.5627 \mathrm{e} 0$ & $2.0948 \mathrm{e} 0$ & $4.4351 \mathrm{e}-1$ & $5.0859 \mathrm{e}-2$ \\
2 & $2.4310 \mathrm{e} 0$ & $2.3886 \mathrm{e} 0$ & $2.3037 \mathrm{e} 0$ & $1.8812 \mathrm{e} 0$ & $4.8645 \mathrm{e}-1$ & $5.4931 \mathrm{e}-2$ \\
3 & $2.3129 \mathrm{e} 0$ & $2.2749 \mathrm{e} 0$ & $2.1802 \mathrm{e} 0$ & $1.8076 \mathrm{e} 0$ & $4.9837 \mathrm{e}-1$ & $6.4541 \mathrm{e}-2$ \\
4 & $2.6706 \mathrm{e} 0$ & $2.6225 \mathrm{e} 0$ & $2.5193 \mathrm{e} 0$ & $2.0636 \mathrm{e} 0$ & $5.8780 \mathrm{e}-1$ & $9.2958 \mathrm{e}-2$ \\
5 & $3.1593 \mathrm{e} 0$ & $2.9673 \mathrm{e} 0$ & $2.8141 \mathrm{e} 0$ & $2.2948 \mathrm{e} 0$ & $6.2242 \mathrm{e}-1$ & $9.8928 \mathrm{e}-2$ \\
6 & $2.7198 \mathrm{e} 0$ & $2.5764 \mathrm{e} 0$ & $2.4233 \mathrm{e} 0$ & $1.9427 \mathrm{e} 0$ & $5.3022 \mathrm{e}-1$ & $7.5071 \mathrm{e}-2$ \\
7 & $2.9581 \mathrm{e} 0$ & $2.8158 \mathrm{e} 0$ & $2.6886 \mathrm{e} 0$ & $2.2162 \mathrm{e} 0$ & $6.1367 \mathrm{e}-1$ & $9.9160 \mathrm{e}-2$ \\
8 & $2.9712 \mathrm{e} 0$ & $2.8012 \mathrm{e} 0$ & $2.6446 \mathrm{e} 0$ & $2.1981 \mathrm{e} 0$ & $6.4002 \mathrm{e}-1$ & $9.3180 \mathrm{e}-2$ \\
9 & $3.7158 \mathrm{e} 0$ & $3.2765 \mathrm{e} 0$ & $3.0548 \mathrm{e} 0$ & $2.4382 \mathrm{e} 0$ & $6.8837 \mathrm{e}-1$ & $1.1892 \mathrm{e}-1$ \\
10 & $3.1307 \mathrm{e} 0$ & $2.7671 \mathrm{e} 0$ & $2.5808 \mathrm{e} 0$ & $2.0749 \mathrm{e} 0$ & $5.9963 \mathrm{e}-1$ & $8.4462 \mathrm{e}-2$ \\
11 & $3.0937 \mathrm{e} 0$ & $2.8429 \mathrm{e} 0$ & $2.6748 \mathrm{e} 0$ & $2.1673 \mathrm{e} 0$ & $5.7858 \mathrm{e}-1$ & $8.8655 \mathrm{e}-2$ \\
12 & $3.1317 \mathrm{e} 0$ & $2.7967 \mathrm{e} 0$ & $2.6259 \mathrm{e} 0$ & $2.1068 \mathrm{e} 0$ & $5.8031 \mathrm{e}-1$ & $8.6055 \mathrm{e}-2$ \\
\hline
\end{tabular}

If higher accuracy is pursued, we can take more correction steps at the finest level $k=q$. See Figure 5 for the convergence history of both the gamblet based method and the geometric multigrid based method up to $10^{-14}$. The gamblet based method converges much faster than the geometric multigrid based method.
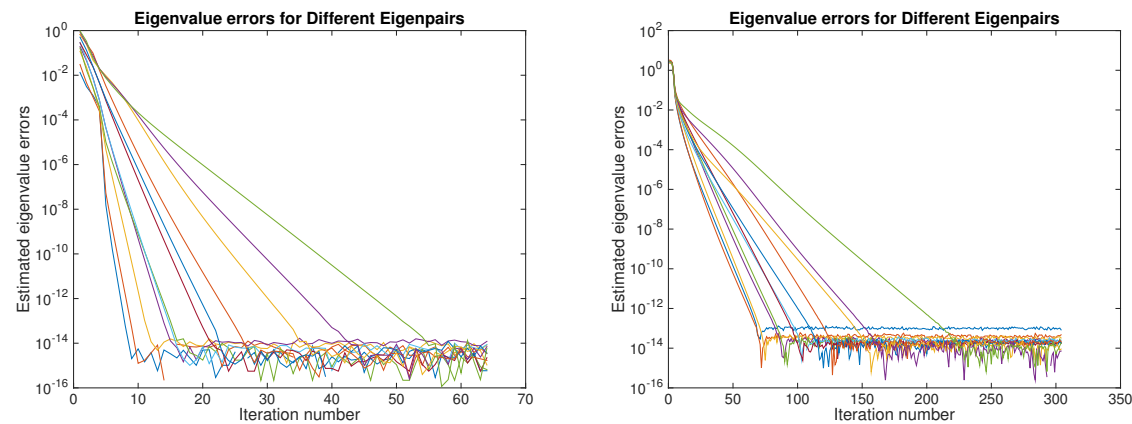

FIG. 5. Convergence history for first 12 eigenvalues. Left: Gamblet based multilevel method. Right: Geometric mutligrid based multilevel method. The iteration number corresponds to the number of correction steps, namely the outer iteration number. The first a few iterations are on the coarse levels $k=3, \ldots, q-1$, and the following iterations are on the finest level $k=q$.

Copyright $@$ ㅇ by SIAM. Unauthorized reproduction of this article is prohibited. 


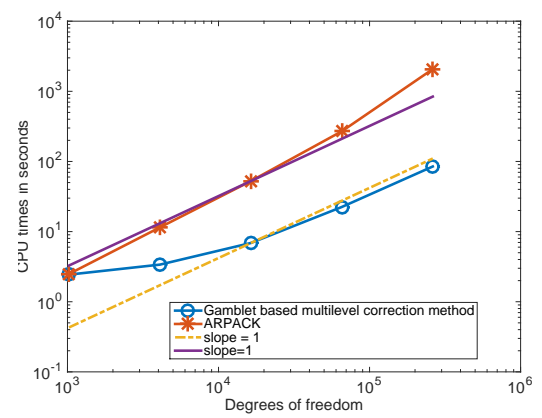

FIG. 6. CPU time for Gamblet based multilevel correction and ARPACK.

We now compare the efficiency of the multilevel correction method with the benchmark solver ARPACK (https://www.caam.rice.edu/software/ARPACK/). We implement the multilevel correction method in $\mathrm{C}$ (with a precomputed Gamblet decomposition), and run the code on a machine with two 6-core dual thread Intel Xeon E5-2620 2.00GHz CPUs with 72GB memory. We solve for 12 eigenvalues, and stop the multilevel correction method when relative errors for all eigenvalues are below $10^{-9}$. For comparison, we use the ARPACK library to solve the same eigenvalue problems, and use the geometric multigrid method to solve the corresponding linear systems. The results in Figure 6 show that the Gamblet based multilevel correction method achieves a ten-fold acceleration in terms of CPU time. We only plot the "online" computing time for eigenpairs in Figure 6, the "offline" precomputing time for the gamblet decomposition is not included since we only have a MATLAB implementation for this part. For the MATLAB implementation of the multilevel correction method, the running time for the "online" and "offline" parts are usually proportional, and the a priori theoretical bound on the complexity of the gamblets precomputation is $\mathcal{O}\left(N \ln ^{2 d+1} N\right)$.

4.2. Random checkerboard. In the second example, we consider the eigenvalue problem for the random checkerboard case. Here, $\Omega=[-1,1] \times[-1,1]$ and the matrix $a(x)$ is a realization of random coefficients taking values 20 or $1 / 20$ with probability $1 / 2$ at small scale $\varepsilon=1 / 64$; see Figure 7 . The coefficient $a(x)$ has contrast $4 \times 10^{2}$, and is highly oscillatory.

We calculate the first 12 eigenvalues. The parameters for Algorithm 3.2 are $H=1 / 2, q=7$, and we take $\mathfrak{V}^{(2)}$ as the coarsest subspace. We choose $m_{1}=m_{2}=2$ and $p=1$, and use Gauss-Seidel as the smoother in Algorithm 2.2. We take the number of subspace correction steps $\varpi=1$ for $k=3, \ldots, q-1$, then we run the subspace correction at the finest level $k=q$ until convergence.

The convergence rates shown in Figure 8 suggest a ten-fold acceleration in terms of iteration number when comparing the gamblet and based multilevel correction method to the geometric multigrid based multilevel correction method. While it takes more than 800 iterations for geometric multigrid based multilevel correction method to 


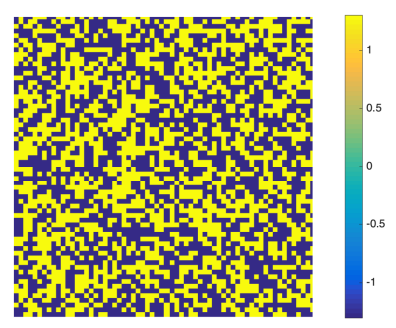

FIG. 7. Random Checkerboard coefficient, in $\log _{10}$ scale.

converge for the first 12 eigenvalues to converge to accuracy $10^{-14}$, the gamblet based multilevel correction method converges to that accuracy within 70 outer iterations.
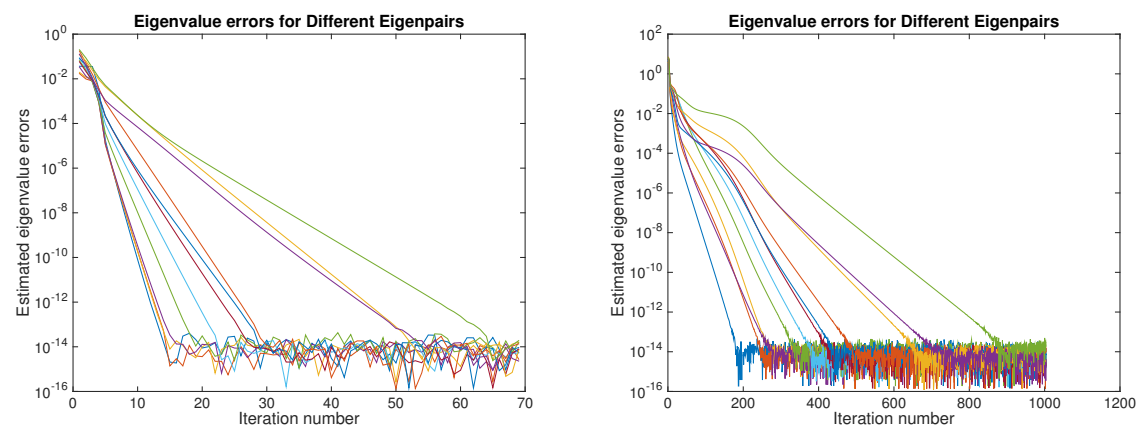

FIG. 8. Convergence history for first 12 eigenvalues. Left: gamblet based method. Right: geometric mutligrid based method. The iteration number corresponds to the number of correction steps, namely, the outer iteration number. The first a few iterations are on the coarse level $k=$ $3, \ldots, q-1$, and the following iterations are on the finest level $k=q$.

4.3. Gamblet preconditioned LOBPCG method. In the previous sections, we have proposed the Gamblet based multilevel correction scheme, proved its convergence and numerically demonstrated its performance. In this section, we will show that Gamblets can also be used as an efficient preconditioner for existing eigensolvers. To be precise, we construct the gamblet based preconditioner for the Locally Optimal Block Preconditioned Conjugate Gradient (LOBPCG) method [25, 28], which is a class of widely used eigensolvers.

A variety of Krylov subspace-based methods are designed to solve a few extreme eigenvalues of symmetric positive matrix $[49,19,10,24,25,28,7]$. Many studies have shown that LOBPCG is one of the most effective method at this task $[27,18]$ and there are various recent developments of LOBPCG for indefinite eigenvalue problems [8], nonlinear eigenvalue problems [51], electronic structure calculation [52], and tensor decomposition [46]. The main advantages of LOBPCG are that the costs per iteration and the memory use are competitive with those of the Lanczos method, linear convergence is theoretically guaranteed and practically observed, it allows utilizing highly efficient matrix-matrix operations, e.g., BLAS 3, and it can directly take advantage of preconditioning, in contrast to the Lanczos method.

LOBPCG can be seen as a generalization of the Preconditioned Inverse Iteration (PINVIT) method [19, 10, 24]. The PINVIT method [19, 10, 24, 25, 28], can be 
motivated as an inexact Newton method for the minimization of the Rayleigh quotient. The Rayleigh quotient $\mu(x)$ for a vector $x$ and a symmetric, positive definite matrix $M$ is defined by

$$
\mu(x, M):=\mu(x)=\frac{x^{T} M x}{x^{T} x} .
$$

The global minimum of $\mu(x)$ is achieved at $x=v_{1}$, with $\lambda_{1}=\mu(x)$, where $\left(\lambda_{1}, v_{1}\right)$ is the eigenvalue pair of $M$ corresponding to the smallest eigenvalue $\lambda_{1}$. This means that minimizing the Rayleigh quotient is equal to computing the smallest eigenvalue. With the following inexact Newton method, from

$$
\begin{aligned}
w_{i} & =B^{-1}\left(M x_{i}-\mu\left(x_{i}\right) x_{i}\right), \\
x_{i+1} & =x_{i}-w_{i},
\end{aligned}
$$

we get the PINVIT. The preconditioner $B$ for $M$ have to satisfy $\left\|I-B^{-1} M\right\|_{M} \leq$ $c<1$. The inexact Newton method can be relaxed by adding a step size $\alpha$

$$
x_{i+1}=x_{i}-\alpha_{i} w_{i}
$$

Finding the optimal step size $\alpha_{i}$ is equivalent to solving the a small eigenvalue problem with respect to $M$ in the subspace $\left\{x_{i}, w_{i}\right\}$. In [25] Knyazev used the optimal vector in the subspace $\left\{x_{i-1}, w_{i}, x_{i}\right\}$ as the next iterate. The resulting method is called LOBPCG.

In the following comparison, we adopt the MATLAB implementation of LOBPCG by Knyazev [26]. We use the gamblet based multigrid as a preconditioner in the LOBPCG method, and compare its performance for SPE10 example with geometric multigrid preconditioned CG (GMGCG) and general purpose ILU based preconditioner; the results are shown in Figure 9. It is clear that the gamblet preconditioned LOBPCG as well as the gamblet multilevel correction scheme (see Figure 9) have better performance than the GMGCG or ILU preconditioned LOBPCG in terms of iteration number. The gamblet based LOBPCG converges with the accuracy (residuals) of about $10^{-15}$, with 56 iterations in about 30 seconds (in addition, the precomputation of the gamblets costs about 18 seconds). While the GMG preconditioned LOBPCG in Figure 9 fails to converge in 1000 iterations, and the residuals are above $10^{-5}$ when it is stopped at 1000 iterations in about 60 seconds. Although our implementation in MATLAB is not optimized in terms of speed, the above observations indicate that the gamblet preconditioned LOBPCG has the potential to achieve even better performance with an optimized implementation.

Remark 4.2. The LOBPCG method has a larger subspace for the small RayleighRitz eigenvalue problem, compared with the multilevel correction scheme in (3.37). This could be the reason why the gamblet preconditinoed LOBPCG scheme has fewer (but comparable) outer iterations compared with the multilevel correction scheme shown in Figure 5. On the other hand, orthogonalization is crucial for a robust implementation of LOBPCG, and adaptive stopping criteria needs to be used for efficiency. Delicate strategies [18] are proposed in order to ensure the robustness of LOBPCG. Comparing with LOBPCG, the gamblet based multilevel correction scheme appears to be very robust in our numerical experiments: we only solve an eigenvalue problem at the coarsest level, and still achieve an accuracy of $10^{-14}$ without using any adaptive stopping criteria; for example, see Figure 5. 


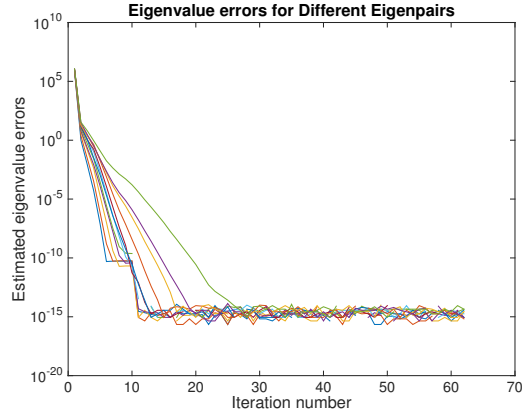

(a) Eigenvalue errors for the gamblet preconditioned LOBPCG.

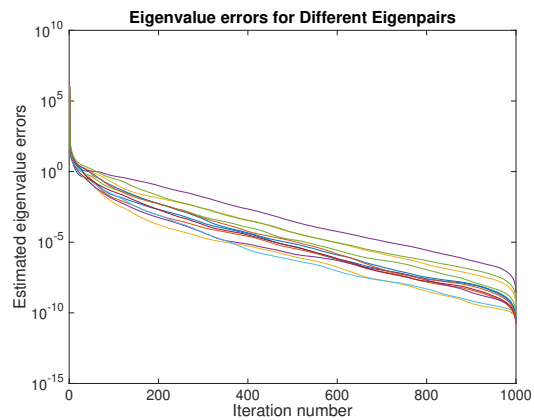

(c) Eigenvalue errors for the GMGCG preconditioned LOBPCG.

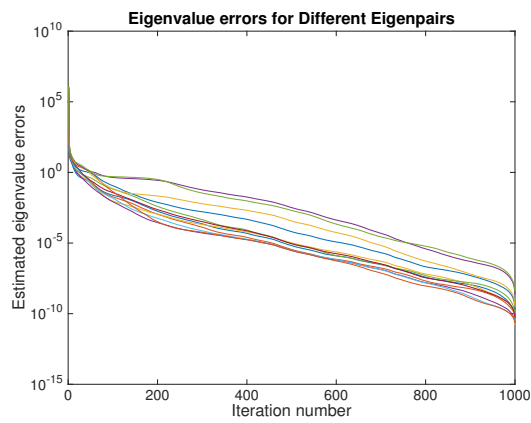

(e) Eigenvalue errors for ILU preconditioned LOBPCG.

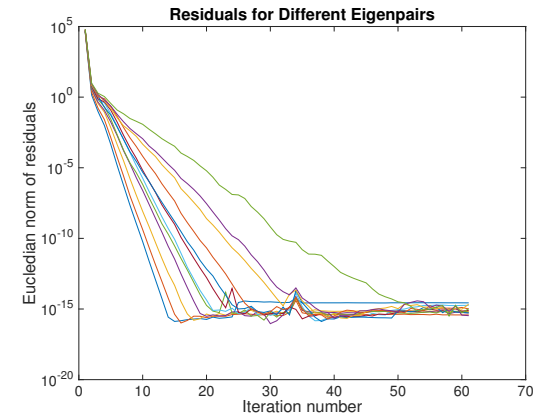

(b) Residuals for the gamblet preconditioned LOBPCG.

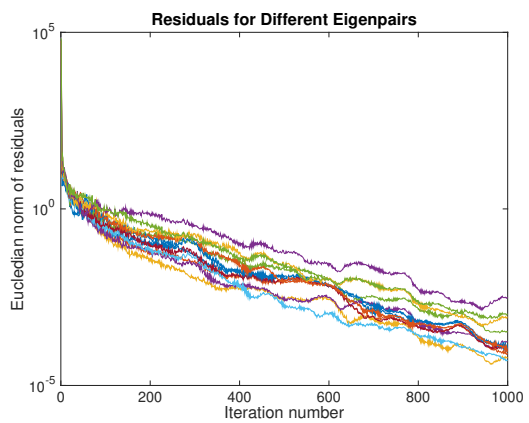

(d) Residuals for the GMGCG preconditioned LOBPCG.

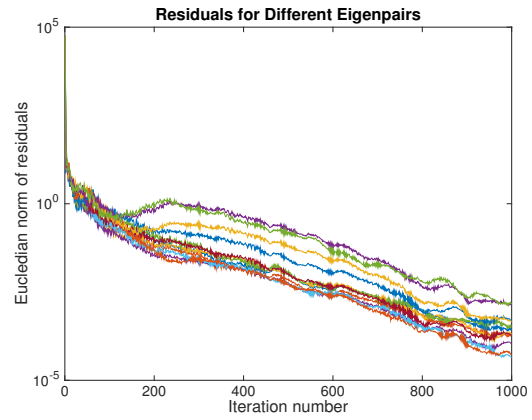

(f) Residuals for ILU preconditioned LOBPCG.

FIG. 9. Eigenvalue errors and residuals for the first 12 eigenpairs of the eigenvalue problems for SPE 10 case. Top row: gamblet preconditioned LOBPCG. Middle row: geometric multigrid preconditioned LOBPCG. Bottom row: ILU preconditioned LOBPCG (using MATLAB command ichol( $A$, struct('michol', 'on'))).

Combination of multilevel correction with LOBPCG. We noticed that a "good"

Copyright (c) by SIAM. Unauthorized reproduction of this article is prohibited. 
initial value is important for the convergence of the LOBPCG method. Therefore, we propose combining the multilevel correction scheme and LOBPCG to derive a hybrid method. In this combination, the gamblet based multilevel correction scheme is used to compute, to a high accuracy, an initial approximation for the eigenpairs for the gamblet preconditioned LOBPCG scheme. We use this combined method to solve the so-called Anderson Localization eigenvalue problem in the following subsection. Since LOBPCG is based on the so-called Ky Fan trace minimization principle, at each step the sum of the eigenvalues are minimized [32]. Therefore, the convergence rate of different eigenvalues will be balanced.

4.3.1. Anderson localization. Consider the linear Schrödinger operator $\mathcal{H}:=$ $-\Delta+V(x)$ with disorder potential $V(x)$ (as presented in [2]) whose Anderson localization [3] properties are analyzed in [4] and in [2] (see [9] and references therein for the ubiquity and importance of localization in wave physics).

Let $\Omega:=[-1,1]^{2}$ be the domain of the operator. By [2], $V(x)$ is a disorder potential that vary randomly between two values $\beta \geq \frac{1}{\varepsilon^{2}} \gg \alpha$ on a small scale $\varepsilon$. In the numerical experiment, we choose $\varepsilon=0.01, \beta=10^{4}$, and $\alpha=1$ (the eigenvalue problem becomes more difficult as $\varepsilon$ becomes smaller). See Figure 10 for results using the gamblet based multilevel correction method, gamblet preconditioned LOBPCG, and the hybrid method.
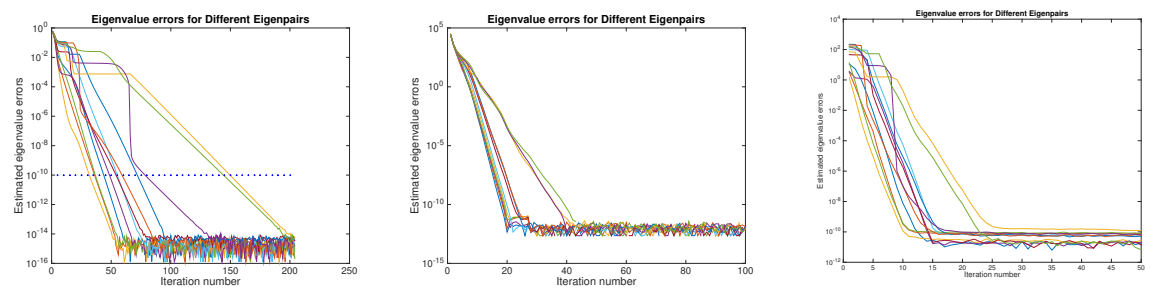

FIG. 10. Convergence history for the first 12 eigenvalues. Left: using the gamblet based multilevel correction method. Middle: using the gamblet preconditioned LOBPCG method. Right: using the hybrid method, namely, generating the initial approximation by the gamblet based multilevel method, then preforming the gamblet preconditioned LOBPCG method until convergence. The iteration number corresponds to the number of correction steps, namely, the outer iteration number. The first few iterations are on the coarse level $k=3, \ldots, q-1$, and the following iterations are on the finest level $k=q$.

Acknowledgments. We thank Florian Schaefer for stimulating discussions. We thank two anonymous reviewers whose comments have greatly improved this manuscript.

\section{REFERENCES}

[1] R. A. Adams and J. Fournier, Sobolev Spaces, 2nd ed., Pure Appl. Math. (Amst.) 140, Elsevier/Academic Press, Amsterdam, 2003.

[2] R. Altmann, P. Henning, And D. Peterseim, Quantitative Anderson Localization of Schrödinger Eigenstates under Disorder Potentials, https://arxiv.org/abs/1803.09950, 2018.

[3] P. W. Anderson, Absence of diffusion in certain random lattices, Phys. Rev., 109 (1958), pp. 1492-1505.

[4] D. N. Arnold, G. David, D. Jerison, S. Mayboroda, and M. Filoche, Effective confining potential of quantum states in disordered media, Phys. Rev. Lett., 116 (2016), 056602.

[5] I. BABUŠKA AND J. OsBoRN, Finite element-Galerkin approximation of the eigenvalues and eigenvectors of selfadjoint problems, Math. Comp., 52 (1989), pp. 275-297. 
[6] I. BAbušKa And J. Osborn, Eigenvalue problems, in Handbook of Numerical Analysis, Vol. II, Finite Element Methods (Part 1), P. G. Lions and C. P.G., eds., North-Holland, Amsterdam, 1991, pp. 641-787.

[7] Z. Bai, J. Demmel, J. Dongarra, A. Ruhe, and H. van der Vorst, eds., Templates for the Solution of Agebraic Eigenvalue Problems: A Practical Guide, SIAM, Philadelphia, 2000, https://doi.org/10.1137/1.9780898719581.

[8] Z. BAI AND R. C. LI, Minimization principles for the linear response eigenvalue problem I: Theory, SIAM J. Matrix Anal. Appl., 33 (2012), pp. 1075-1100, https://doi.org/10.1137/ 110838960.

[9] J. Billy, V. Josse, Z. Zuo, A. Bernard, B. Hambrecht, P. Lugan, D.Clément, L. Sanchez-Palencia, P. Bouyer, and A. Aspect, Direct observation of Anderson localization of matter waves in a controlled disorder, Nature, 453 (2008), pp. 891-894.

[10] J. Bramble, J. Pasciak, And A. Knyazev, A subspace preconditioning algorithm for eigenvector/eigenvalue computation, Adv. Comput. Math., 6 (1996), pp. 159-189.

[11] A. Brandt, Multi-level Adaptive Technique (MLAT) for Fast Numerical Solutions to Boundary Value Problems, in Proceedings of the 3rd International Conference on Numerical Methods in Fluid Mechanics, Lecture Notes in Physics 18, 1973.

[12] S. C. Brenner, P. Monk, And J. Sun, CO IPG Method for Biharmonic Eigenvalue Problems, in Academy of Mathematics and Systems Science, CAS Colloquia \& Seminars, 2014.

[13] M. E. BRewster AND G. BeYlkin, A multiresolution strategy for numerical homogenization, Appl. Comput. Harmon. Anal., 2 (1995), pp. 327-349.

[14] L. CAO AND J. CUI, Asymptotic expansions and numerical algorithms of eigenvalues and eigenfunctions of the Dirichlet problem for second order elliptic equations in perforated domains, Numer. Math., 96 (2004), pp. 525-581.

[15] F. Chatelin, Spectral Approximation of Linear Operators, Academic Press, New York, 1983.

[16] H. Chen, H. XIE, And F. Xu, A full multigrid method for eigenvalue problems, J. Comput. Phys, 322 (2016), pp. 747-759.

[17] M. Dorobantu And B. Engquist, Wavelet-based numerical homogenization, SIAM J. Numer. Anal., 35 (1998), pp. 540-559, https://doi.org/10.1137/S0036142996298880.

[18] J. A. Duersch, M. Shao, C. Yang, and M. Gu, A robust and efficient implementation of LOBPCG, SIAM J. Sci. Comput., 40 (2018), pp. C655-C676, https://doi.org/10.1137/ $17 \mathrm{M} 1129830$.

[19] E. G. D'yakonov and M. Y. Orekhov, Minimization of the computational labor in determining the first eigenvalues of differential operators, Math. Notes, 27 (1980), pp. 382-391.

[20] W. Hackbusch, A fast iterative method for solving Poisson's equation in a general region, in Numerical Treatment of Differential Equations (Proc. Conf., Math. Forschungsinst., Oberwolfach, 1976), Lecture Notes in Math. 631, Springer, Berlin, 1978, pp. 51-62.

[21] T. Y. Hou, D. Huang, K. C. Lam, and Z. Zhang, A Fast Hierarchically Preconditioned Eigensolver Based on Multiresolution Matrix Decomposition, https://arxiv.org/abs/1804. 03415, 2018.

[22] T. J. R. Hughes, G. R. Feisóo, L. Mazzei, And J.-B. Quincy, The variational multiscale method-A paradigm for computational mechanics, Comput. Methods Appl. Mech. Engrg., 166 (1998), pp. 3-24.

[23] S. JiA, H. XIE, M. XIE, AND F. XU, A full multigrid method for nonlinear eigenvalue problems, Sci. China Math., 59 (2016), pp. 2037-2048.

[24] A. Knyazev, Preconditioned eigensolvers - An oxymoron?, Electron. Trans. Numer. Anal., 7 (1998), pp. 104-123.

[25] A. V. KNYAZEV, Toward the optimal preconditioned eigensolver: Locally optimal block preconditioned conjugate gradient method, SIAM J. Sci. Comput., 23 (2001), pp. 517-541, https://doi.org/10.1137/S1064827500366124.

[26] A. KNYAZEV, LOBPCG.m, https://www.mathworks.com/matlabcentral/fileexchange/ 48-lobpcg-m, 2015, version 1.5.

[27] A. Knyazev, Recent Implementations, Applications, and Extensions of the Locally Optimal Block Preconditioned Conjugate Gradient Method (LOBPCG), https://arxiv.org/abs/ 1708.08354, 2017.

[28] A. KNyAzev AND K. NeymeyR, Efficient solution of symmetric eigenvalue problems using multigrid preconditioners in the locally optimal block conjugate gradient method, Electron. Trans. Numer. Anal., 15 (2003), pp. 38-55.

[29] W. Konn, Analytic properties of Bloch waves and Wannier functions, Phys. Rev. (2), 115 (1959), pp. 809-821.

Copyright (c) by SIAM. Unauthorized reproduction of this article is prohibited. 
[30] R. Kornhuber, D. Peterseim, and H. Yserentant, An analysis of a class of variational multiscale methods based on subspace decomposition, Math. Comp., 87 (2018), pp. 27652774 .

[31] R. Kornhuber and H. Yserentant, Numerical homogenization of elliptic multiscale problems by subspace decomposition, Multiscale Model. Simul., 14 (2016), pp. 1017-1036, https: //doi.org/10.1137/15M1028510.

[32] D. Kressner, M. Pandur, And M. Shao, An indefinite variant of LOBPCG for definite matrix pencils, Numer. Algorithms, 66 (2014), pp. 681-703.

[33] R. Kyng and S. Sachdeva, Approximate Gaussian elimination for Laplacians: Fast, sparse, and simple, in Proceedings of the 57th Annual IEEE Symposium on Foundations of Computer Science, FOCS 2016, IEEE Computer Soc., Los Alamitos, CA, 2016, pp. 573-582.

[34] Q. Lin AND H. XIE, An observation on Aubin-Nitsche lemma and its applications, Math. Pract. Theory, 41 (2011), pp. 247-258.

[35] Q. Lin AND H. XIE, A multilevel correction type of adaptive finite element method for Steklov eigenvalue problems, in Proceedings of the International Conference Applications of Mathematics, 2012, pp. 134-143.

[36] Q. Lin AND H. XIE, A multi-level correction scheme for eigenvalue problems, Math. Comp., 84 (2015), pp. 71-88.

[37] A. MÅlqvist and D. Peterseim, Computation of eigenvalues by numerical upscaling, Numer. Math., 130 (2014), pp. 337-361, https://doi.org/10.1007/s00211-014-0665-6.

[38] A. Målqvist and D. Peterseim, Localization of elliptic multiscale problems, Math. Comp., 83 (2014), pp. 2583-2603.

[39] C. A. Micchelli and T. J. Rivlin, A survey of optimal recovery, in Optimal Estimation in Approximation Theory, Plenum, New York, 1977, pp. 1-54.

[40] M. Olshanskit And E. Tyrtyshnikov, Iterative Methods for Linear Systems: Theory and Applications, SIAM, Philadelphia, 2014, https://doi.org/10.1137/1.9781611973464.

[41] H. OWHADI, Multigrid with rough coefficients and multiresolution operator decomposition from hierarchical information games, SIAM Rev., 59 (2017), pp. 99-149, https://doi.org/10. $1137 / 15 \mathrm{M} 1013894$.

[42] H. Owhadi And C. Scovel, Universal Scalable Robust Solvers from Computational Information Games and Fast Eigenspace Adapted Multiresolution Analysis, https://arxiv.org/abs/ 1703.10761, 2017.

[43] H. Owhadi And C. Scovel, Operator Adapted Wavelets, Fast Solvers, and Numerical Homogenization From a Game Theoretic Approach to Numerical Approximation and Algorithm Design, Cambridge Monographs on Applied and Computational Mathematics, Cambridge University Press, Cambridge, 2019.

[44] H. OWHADI AND L. ZHANG, Gamblets for opening the complexity-bottleneck of implicit schemes for hyperbolic and parabolic ODEs/PDEs with rough coefficients, J. Comput. Phys., 347 (2017), pp. 99-128.

[45] H. Owhadi, L. Zhang, and L. Berlyand, Polyharmonic homogenization, rough polyharmonic splines and sparse super-localization, ESAIM Math. Model. Numer. Anal., 48 (2014), pp. 517-552, https://doi.org/10.1051/m2an/2013118.

[46] M. Rakhuba and I. Oseledets, Calculating vibrational spectra of molecules using tensor train decomposition, J. Chem. Phys., 145 (2016), 124101.

[47] Y. SAAD, Numerical Methods For Large Eigenvalue Problems, SIAM, Philadelphia, 2011, https: //doi.org/10.1137/1.9781611970739.

[48] F. Schäfer, T. J. Sullivan, And H. Owhadi, Compression, Inversion, and Approximate PCA of Dense Kernel Matrices at Near-Linear Computational Complexity, https://arxiv.org/ abs/1706.02205, 2017.

[49] D. Sorensen, Implicitly Restarted Arnoldi/Lanczos Methods for Large Scale Eigenvalue Calculations, Kluwer Acad. Publ., Dordrecht, 1997.

[50] G. Strang And G. Fix, An Analysis of the Finite Element Method, Prentice-Hall, Englewood Cliffs, NJ, 1973.

[51] D. B. Szyld And F. Xue, Preconditioned eigensolvers for large-scale nonlinear Hermitian eigenproblems with variational characterizations. I. External eigenvalues, Math. Comp., 85 (2016), pp. 2887-2918.

[52] E. Vecharynski, C. YAng, And J. E.PASk, A projected preconditioned conjugate gradient algorithm for computing many extreme eigenpairs of a Hermitian matrix, J. Comput. Phys., 290 (2015), pp. 73-89.

[53] W. L. Wan, T. F. Chan, And B. Smith, An energy-minimizing interpolation for robust multigrid methods, SIAM J. Sci. Comput., 21 (1999/00), pp. 1632-1649, https://doi.org/10. $1137 / \mathrm{S} 1064827598334277$.

Copyright $@$ by SIAM. Unauthorized reproduction of this article is prohibited. 
[54] G. H. Wannier, Dynamics of band electrons in electric and magnetic fields, Rev. Modern Phys., 34 (1962), pp. 645-655.

[55] H. XIE, A multigrid method for eigenvalue problem, J. Comput. Phys., 274 (2014), pp. 550-561.

[56] H. XIE, A type of multilevel method for the Steklov eigenvalue problem, IMA J. Numer. Anal., 34 (2014), pp. 592-608.

[57] J. Xu And A. Zhou, A two-grid discretization scheme for eigenvalue problems, Math. Comp., 70 (2001), pp. 17-25.

[58] G. R. Yoo AND H. OwHADI, De-noising by thresholding operator adapted wavelets, Stat. Comput., in press, https://doi.org/10.1007/s11222-019-09893-x.

[59] L. Zhang, L. CAO, AND X. WANG, Multiscale finite element algorithm of the eigenvalue problems for the elastic equations in composite materials, Comput. Methods Appl. Mech. Engrg., 198 (2009), pp. 2539-2554.

[60] S. Zhang, Y. XI, AND X. Ji, A multi-level mixed element method for the eigenvalue problem of biharmonic equation, J. Sci. Comput., 75 (2018), pp. 1415-1444.

Copyright (c) by SIAM. Unauthorized reproduction of this article is prohibited. 